\title{
A Model of the Effects of Fluid Variation due to Body Position on Cheyne-Stokes Respiration
}

\author{
by \\ Marianne Wilcox
}

\begin{abstract}
A Thesis
presented to

The University of Guelph
\end{abstract}

In partial fulfilment of requirements

for the degree of

Master of Science

in

Mathematics

Guelph, Ontario, Canada

(c) Marianne Wilcox, January, 2013 


\begin{abstract}
A model of the effects of fluid variation due to body position on Cheyne-Stokes respiration

Advisors:

Marianne Wilcox

University of Guelph, 2013

Dr. Allan R. Willms

Dr. William F. Langford
\end{abstract}

Cheyne-Stokes respiration is a distinct breathing pattern consisting of periods of hyperpnea followed by apneas, with unknown etiology. One in two patients with congestive heart failure suffer from this condition. Researchers hypothesize that key factors in CSR are the fluid shift from the standing to supine position and the differences between genders. A mathematical model of the cardio-respiratory system was constructed using parameter values from real data. Hopf bifurcation analysis was used to determine regions of stable versus oscillatory breathing patterns. In the model, Cheyne-Stokes respiration is more likely to occur while in the supine position and males are more likely to develop Cheyne-Stokes than females. These findings, which are in agreement with clinical experience, suggest that both gender and fluid shift contribute to the pathogenesis of Cheyne-Stokes respiration, and that physical quantities such as blood volumes and neural feedback may be sufficient to explain the observations of CSR. 


\section{Acknowledgments}

I would like to thank first and foremost my advisor, Allan Willms, for persevering with me throughout all endeavours during my graduate career thus far. His unwavering commitment, dedication, and knowledge made this entire process one of my greatest

achievements; thank you. I would also like to thank William Langford, his guidance through my thesis process does not go unrecognized; my sincerest thanks.

I would like to thank Dr. T. D. Bradley, Azadeh Yadollahi and everyone in the Sleep Lab at the Toronto Rehabilitation Institute for the data, access to the lab and the idea of studying the effects of the supine position.

I would like to thank my family. Mom and Dad have always exemplified what it means to be dedicated. Their continual and unconditional love, support, and affection surround everything I do. Ben's assurance and determination to BE the man he IS, regardless of society or others opinions is inspiring. Rebecca's (Boogie) persistence for finding laughter in every walk of life, and her infectious positive outlook on all things is contagious.

I would like to especially thank Jon Waito, for accepting nothing but perfection from me. He always pushes and guides me to be the best I can be; he is my rock.

I would like to thank the University of Guelph, especially The Department of Mathematics and Statistics. To all faculty and staff who made this degree possible.

Lastly, I would like to thank my fellow graduate students - those who have moved on, those still in the trenches, and those just beginning - for their support, feedback, and most of all, friendship. 


\section{Contents}

Acknowledgments $\quad$ iii

1 Overview 1

2 Dong and Langford Model 5

2.1 Model Description . . . . . . . . . . . . . . . 5

2.1.1 Ventilation-Perfusion Ratio . . . . . . . . . . . 7

2.1 .2 Gain ..................... 7

2.2 Hopf Bifurcation . . . . . . . . . . . . . . . . . . 7

3 Sensitivity Analysis $\quad 10$

3.1 Increasing and Decreasing Parameters By $30 \%$. . . . . . . . . . 10

3.2 Constant Area . . . . . . . . . . . . . . . . . . . . . . . 11

4 Body Mass Model 15

4.1 Introduction: Obesity . . . . . . . . . . . . . . 15

4.2 Model Adjustments . . . . . . . . . . . . . . . . . . . . . . . . 15

4.2.1 Body Size Adjustments . . . . . . . . . . . . . . . . . . 15

4.2 .2 Obesity Adjustments . . . . . . . . . . . . . . 16

4.3 Results.............................. 18

4.3 .1 Body Size . . . . . . . . . . . . . . . 18

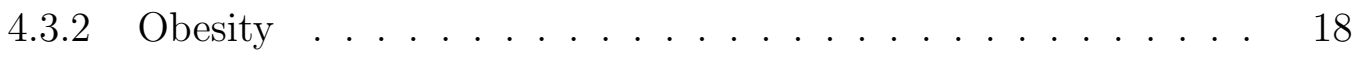

4.4 Conclusion . . . . . . . . . . . . . . . . . . . . 20 
5 Fluid Shift Model $\quad 22$

5.1 Introduction: Fluid Shift . . . . . . . . . . . . . . . 22

5.2 Mathematical Model . . . . . . . . . . . . . . . . 23

5.2.1 Circulatory System ................... 23

5.2.2 Respiratory System . . . . . . . . . . . . . . . . 28

5.2.3 Control System . . . . . . . . . . . . . . . . . . . . 29

5.2 .4 Model Equations . . . . . . . . . . . . . . . . 31

5.3 Nondimensionalized Variables . . . . . . . . . . . . . . . . 32

5.4 Model Analysis . . . . . . . . . . . . . . . . . 36

5.4.1 Hopf Bifurcation ................. 36

5.5 Sensitivity Analysis . . . . . . . . . . . . . . . . . . . . . 39

5.5.1 Increasing and Decreasing Parameters By 30\% . . . . . . . . . 39

5.6 Results............................ 43

5.6.1 Experimental Data .................. 43

5.6.2 Body Position . . . . . . . . . . . . . . . . 44

5.6 .3 Gender....................... 46

5.7 Average Male and Female . . . . . . . . . . . . . . . . . . . 54

6 Conclusions and Future Work $\quad 57$ 


\section{List of Figures}

2.1 (a) steady breathing pattern. (b) oscillatory breathing pattern. . . . . 9

3.1 Sensitivity analysis; $v_{T}, v_{1-7}$ Dong and Langford. . . . . . . . . . . . 12

3.2 Sensitivity analysis; $V_{9}, p_{I}, m$ Dong and Langford. . . . . . . . . . . . 13

4.1 Hopf bifurcation curves for typical adults. . . . . . . . . . . . . 19

4.2 Hopf bifurcation curves for adults with varying body mass. . . . . . . 21

5.1 Schematic diagram of the heart and circulatory system. . . . . . . . . 24

5.2 Hill function. . . . . . . . . . . . . . . . . 30

5.3 Sensitivity analysis; $v_{1-7}$ fluid shift. . . . . . . . . . . . . . . 40

5.4 Sensitivity analysis; $v_{8-14}$ fluid shift. . . . . . . . . . . . . . 41

5.5 Sensitivity analysis; $V_{9}, p_{I}, V_{T}, m$ fluid shift. . . . . . . . . . . . 42

5.6 Hill function corresponding to changes in the Hopf curve. . . . . . . . 47

5.7 Hopf bifurcation curves for male subjects. . . . . . . . . . . . . . . 48

5.8 Fluid shift Hopf curves for male subjects. . . . . . . . . . . . . . . . . 48

5.9 Hopf bifurcation curves for female subjects. . . . . . . . . . . . . 52

5.10 Fluid shift Hopf curves for female subjects. . . . . . . . . . . . . 53

5.11 Hopf curves for average male and female. . . . . . . . . . . . 56 


\section{List of Tables}

3.1 Area between sensitivity analysis curves. . . . . . . . . . . . . . . 14

3.2 Parameter values corresponding to an area of 0.001 between sensitivity analysis curves. . . . . . . . . . . . . . . . . . . 14

5.1 Classification of compartments into body regions. . . . . . . . . . 28

5.2 Solutions to Equation 5.17 for respective $a$ values. . . . . . . . . . . . 34

5.3 Eigenvalues for Subjects 1 to $7 \ldots \ldots$. . . . . . . . . . 37

5.4 Jacobian matrix for Equation 5.16. . . . . . . . . . . . . . . 38

5.5 Area between sensitivity analysis curves. . . . . . . . . . . . . . . . 43

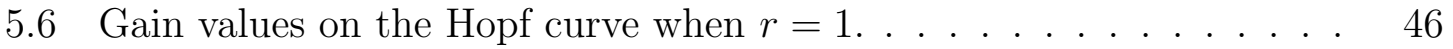

5.7 Male body fluid shift. . . . . . . . . . . . . . . . . . . 50

5.8 Female body fluid shift. . . . . . . . . . . . . . . . 50

6.1 Typical parameters for Dong and Langford model. . . . . . . . . . . . 60

6.2 Typical parameter values for fluid shift model. . . . . . . . . . . . . . 61

6.3 Breakdown of compartmental volumes from total. . . . . . . . . . . 62

6.4 Normalized measured fluid volume. . . . . . . . . . . . . . . 62

6.5 Regional blood flow/Metabolic Rate. . . . . . . . . . . . 63 


\section{Chapter 1}

\section{Overview}

Sleep apnea is a well known sleep disorder characterized by periods of abnormal cessations between breaths or marked reduction in respiratory depth. There are three main classifications of sleep apnea: obstructive (OSA), central (CSA) and a combination of the two (termed mixed or complex sleep apnea). OSA is the most common class of sleep apnea, physiological in nature, in which the pharynx collapses to varying degrees during sleep [13]. CSA, also known as Cheyne-Stokes respiration (CSR), results from neurological conditions that currently are not very well understood [14].

John Cheyne and William Stokes first discovered Cheyne-Stokes Respiration in the early 1800's [36]. CSR is characterized by progressively deeper hyperpneas (increased

depth of breathing) followed by apneas (decrease or stop in breathing) typically occurring during sleep [47]. In normal respiratory control, a negative feedback loop allows oxygen $\left(\mathrm{O}_{2}\right)$ and carbon dioxide $\left(\mathrm{CO}_{2}\right)$ to be supplied and expelled at a constant equilibrium. In essence, Cheyne-Stokes respiration is a consequence of apneas leading to increased $\mathrm{CO}_{2}$ concentrations (because of a delayed transport from the lungs to the chemoreceptors in the neck), causing hyperpneas that overcompensate and decrease $\mathrm{CO}_{2}$ concentrations below the threshold, causing apnea. This series of events gives this condition its cyclical nature of abnormal breathing patterns, the definition of CSR $[22,47]$. Research states that being of the male gender, or suffering from certain medical conditions (congestive heart failure, atrial fibrillation and strokes) 
predispose an individual to an increased likelihood of developing CSR [14, 47]. It is widely known that males are more likely than females to experience CSR $[14,40]$. It is also suggested that 1 in 2 patients suffering from congestive heart failure, suffer from CSR [34].

Many mathematical models have been established throughout the past 70 years in an attempt to better understand CSR. A variety of models of the cardiovascular and respiratory systems have been constructed $[8,9,10,11,16,18,20,24,26,28$, $31,39,42]$. The models that are most related to our research mathematically use Hopf bifurcation theory, which include $[6,15,29]$. The first model ever to use Hopf bifurcation to explain the onset of this disease was constructed by Mackey and Glass in 1977 [29]. They constructed a model of a physiological system using a first-order delay-differential equation. Here, bifurcation theory was used to analyze the model. A similar method of analysis can also be seen in Atamanyk and Langford as well as Dong and Langford $[6,15]$. Hopf bifurcation curves were used to determine the onset of CSR while varying specific parameters [6, 15].

Studies have shown that Cheyne-Stokes respiration is supine dependent [38]. While in the recumbent position cycles of hyperpneas and apneas are more frequent, however the cause is unknown [38]. Recent research has indicated that this supine dependency could be due to the bodily fluid shift experienced upon entering the recumbent position [40]. The theory states that being in an erect position for most of the day, fluid tends to pool in the lower half of the body from gravitational pull. Upon laying down, this fluid shifts back up into the rest of the body, with an excess back-flow into the neck region, contributing to the onset of CSR [40]. Fluid shift has differing responses in males and females; men experience fluid shift much like a river; fluid flows directly from the legs to the neck and settles there. In females, however, fluid flows in the same directional pattern but a large portion pools in the abdomen and thorax due to the high demand for blood supply in the uterus and breasts. Females therefore have 
a decreased proportion of fluid building in their neck.

In order to reduce the inconsistencies evident in what is known about CSR, the effects of obesity (a well known predecessor of congestive heart failure [41]), body position, and gender were evaluated in regard to their effects of fluid shift in the body using a mathematical model. We hypothesize that the shift in bodily fluid into the neck is the main causal mechanism in the onset of CSR. It is known that obesity can significantly increase the risk of congestive heart failure, and so we hypothesize that obesity predisposes an increased risk of developing CSR because of an overload of fluid present in the body $[40,47]$. We also hypothesize that the fluid shift seen from a change in body position (standing to supine) increases the likelihood of oscillatory breathing patterns, promoting the onset of CSR. Lastly, we hypothesize that the increased incidence of CSR in men vs. women [14] exists because of the discrepancies in the types of fluid shift phenomena that occur in each respective gender.

Data was gathered in the Sleep Lab of the Toronto Rehabilitation Institute with the assistance of Dr. Bradley and his team. Measurements were taken from two male subjects and five female subjects in both the standing and supine body positions. Electrodes were placed on multiple body segments along the right side where bioelectrical impedance was used to measure resistances [37]. Resistances were converted to fluid volumes where they were recorded at time zero and after five minutes in the standing position and at time zero and after 90 minutes in the supine position. This data was used to analyze the model in Chapter 5.

The following thesis is divided into four major sections. The first section (Chapter 2) describes previous work and methods of analysis done by Langford and Dong [15]. We then conducted sensitivity analysis on the parameters of this model in Chapter 3. Throughout Chapter 4, we made small alterations to the model produced by Langford and Dong [15] to account for change in body mass. Following this, Chapter 5 describes a new model for fluid shift. The model is based on [15] and is used to 
model fluid shift due to body position, specifically standing to supine. It aims to display regions of oscillatory breathing patterns (CSR) as well as demonstrate the differences in likelihoods between males and females. A full model description along with results and sensitivity analysis are shown. 


\section{Chapter 2}

\section{Dong and Langford Model}

\subsection{Model Description}

Dong and Langford produced a compartmental model of the cardio-respiratory system to determine regions in parameter space in which Cheyne-Stokes Respiration was likely to arise [15]. The system models carbon dioxide $\mathrm{CO}_{2}$ concentrations in the blood which is circulated throughout the heart and circulatory system. This blood transports oxygen, $\mathrm{O}_{2}$, and carbon dioxide, $\mathrm{CO}_{2}$, to and from all organs, tissues and the lungs $[1,22]$. Highly oxygenated blood leaves the lungs and travels to the capillaries in the tissues. Once there, the blood loses $\mathrm{O}_{2}$ and gains $\mathrm{CO}_{2}$, the product of metabolism. Deoxygenated blood travels from the tissues back up to the lungs, where $\mathrm{CO}_{2}$ is removed and $\mathrm{O}_{2}$ is added $[1,15,22]$.

Concentrations of $\mathrm{CO}_{2}$ and $\mathrm{O}_{2}$ in the blood are monitored and controlled by chemoreceptors in the brain $[19,42]$. These chemoreceptors become stimulated during periods of low oxygen or low carbon dioxide levels in the blood (hypoxia or hypocapnia) [1]. Generally speaking, $\mathrm{CO}_{2}$ concentration is the leading factor contributing to breathing control $[15,19]$. Since CSR is primarily an abnormal breathing pattern, the model is concerned with the transport of $\mathrm{CO}_{2}$ rather than $\mathrm{O}_{2}$ concentrations in the blood.

The system consisted of a single closed loop that transported $\mathrm{CO}_{2}$ throughout the body. This loop was divided into 8 compartments; (1) pulmonary veins, (2) 
left heart, (3) aorta, (4) main arteries, (5) arterioles, (6) systemic capillaries, (7) systemic veins, right heart, pulmonary arteries, and (8) pulmonary capillaries. The model assumes that $\mathrm{CO}_{2}$ concentrations into and out of all but two compartments are equal. The exceptions are that changes in $\mathrm{CO}_{2}$ occur due to metabolism in the systemic capillaries and removal in the lungs.

The respiratory system adds an additional compartment corresponding to the alveoli in the lungs. The Hill function is used to model the control which determines how sensitive the system is to changes in $\mathrm{CO}_{2}$ levels in the lungs.

The resulting model equations are:

$$
\begin{aligned}
\frac{d y_{1}}{d s} & =\frac{1}{\hat{v}_{1}}\left(y_{8}-y_{1}\right) \\
\frac{d y_{2}}{d s} & =\frac{1}{\hat{v}_{2}}\left(y_{1}-y_{2}\right) \\
\frac{d y_{3}}{d s} & =\frac{1}{\hat{v}_{3}}\left(y_{2}-y_{3}\right) \\
\frac{d y_{4}}{d s} & =\frac{1}{\hat{v}_{4}}\left(y_{3}-y_{4}\right) \\
\frac{d y_{5}}{d s} & =\frac{1}{\hat{v}_{5}}\left(y_{4}-y_{5}\right) \\
\frac{d y_{6}}{d s} & =\frac{1}{\hat{v}_{6}}\left(y_{5}-y_{6}+\frac{m}{a}\right) \\
\frac{d y_{7}}{d s} & =\frac{1}{\hat{v}_{7}}\left(y_{6}-y_{7}\right) \\
\frac{d y_{8}}{d s} & =\hat{d}\left(y_{7}-y_{8}\right)-\left[\frac{\dot{V}_{A}}{\dot{Q}}\right] \frac{2}{\hat{V}_{9}} \frac{y_{5}^{n}}{1+y_{5}^{n}}\left(y_{8}-\frac{160+8 p_{I}}{a}\right) .
\end{aligned}
$$

The equilibrium points are:

$$
\begin{gathered}
y_{1}^{*}=y_{2}^{*}=y_{3}^{*}=y_{4}^{*}=y_{5}^{*}=y_{8}^{*}=1 \\
y_{6}^{*}=y_{7}^{*}=1+\frac{m}{a}
\end{gathered}
$$

where

$$
a=8 V_{9} \frac{d m}{r}+8 p_{I}+160
$$


Here, $V_{9}$ is the alveolar gas volume and all other parameters are listed in Chapter 5. Typical parameters values for the system can be seen in Appendix A.

\subsubsection{Ventilation-Perfusion Ratio}

By converting the system to dimensionless variables, an important parameter has been introduced to the system; the ventilation-perfusion ratio. Mathematically this

is represented as $\bar{V}_{A} / \dot{Q}$ where $\overline{\dot{V}}_{A}$ is the ventilation (volume of gas the reaches the alveoli per unit time) and $\dot{Q}$ is the perfusion (blood flow rate which reaches the alveoli). Medical literature suggests that typically the ratio should be between 0.2 and 2.1 with a mean of one $[7,15,43]$. A low ratio means that there is a large amount of excess $\mathrm{CO}_{2}$ in the lungs, which is representative of hypoventilation (very slow rate of breathing). Alternatively, a high ratio means that there is a very low level of $\mathrm{CO}_{2}$ in the lungs, which signifies hyperventilation (deep, rapid breathing).

\subsubsection{Gain}

The gain is introduced into the system as feedback control. It is a measure of how sensitive the chemoreceptors are to changes in $\mathrm{CO}_{2}$ concentrations. A Hill function is used as the control to model the response of the chemoreceptors, as it is the best representation of human respiratory response [15, 22, 25, 29]. Little is known about the actual value of the gain, however it can be predicted from the control function, as it is the slope. The gain is also used as a bifurcation parameter. In the model, increasing the value of the gain results in an increased sensitivity to changes in $\mathrm{CO}_{2}$.

\subsection{Hopf Bifurcation}

Hopf bifurcation theory was used to analyze the system of differential equations that represent the cardio-respiratory system. In general, Hopf bifurcation theory states that at a specific fixed point the system will lose stability, giving rise to limit cycles 
when a real parameter of the system varies [33]. These limit cycles are what allow us to determine areas of stable breathing patterns (stable steady state) and areas in which CSR occur (stable periodic solution).

Hopf bifurcations arise under certain conditions. The system of ordinary differential equations (ODEs) must have a real parameter that varies, known as the bifurcation parameter; here the gain, $\mu$, was used as the bifurcation parameter. The system must also have an equilibrium point, and the Jacobian, $J$, must have a pair of purely imaginary (complex conjugate) eigenvalues [33], at a particular value of the bifurcation parameter known as the bifurcation parameter. The normalized equilibrium point of the system is given by $y^{*}$, seen above in Equation 2.2.

Under normal condition, all eigenvalues have negative real part, thus the equilibrium point is asymptotically stable. Further, there exist complex conjugate pairs of eigenvalues. When a given pair, say $\lambda(\mu)$ and $\bar{\lambda}(\mu)$, cross the imaginary axis in the complex eigen-plane as $\mu$ varies through a critical point, say $\mu=\mu_{0}$, limit cycles emerge [33]. Here, $\mu$ values are greater than $\mu_{0}$ and thus the system of ODEs gives rise to a stable, supercritical bifurcation.

Figure 2.1 (a) shows breathing patterns when the bifurcation parameter $\mu=0.03$ lies below the critical value. Oscillations die out, to a stable equilibrium representing typical breathing in healthy subjects. Figure 2.1 (b) shows breathing patterns when the bifurcation parameter $\mu=0.07$ is above the critical value. Here oscillations persist. This is representative of CSR, found typically in subjects with congestive heart failure or those who have suffered a stroke. 


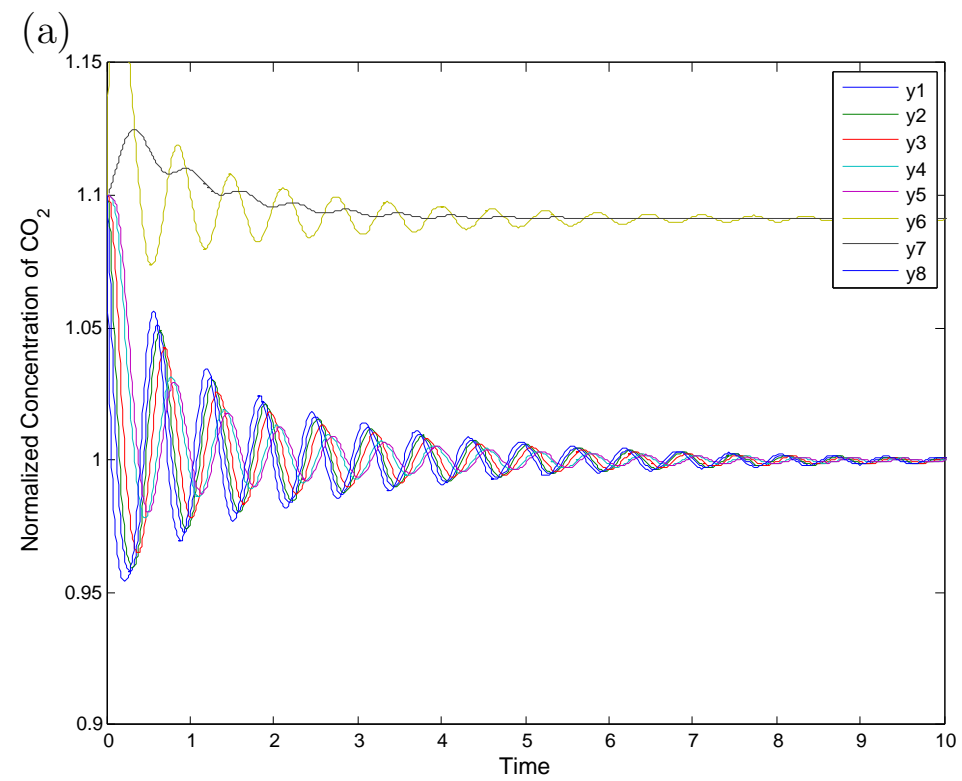

(b)

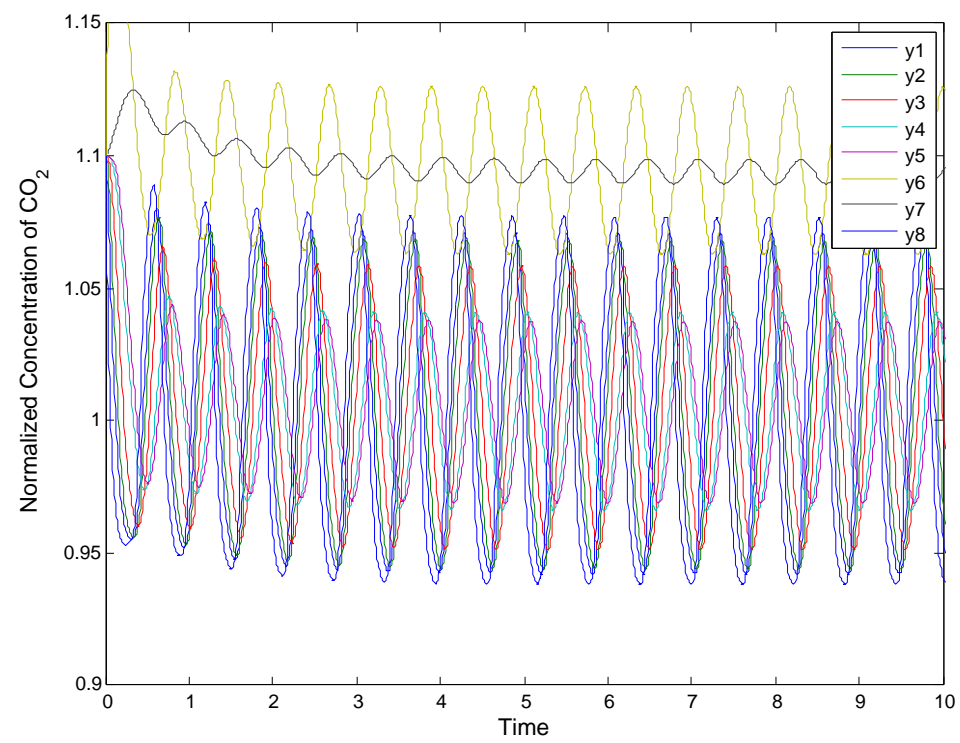

Figure 2.1: Amplitude of a series of breaths seen in (a) steady breathing pattern found in typical subjects; $r=1, \mu=0.03$. (b) oscillatory breathing pattern (CSR); $r=1, \mu=0.07$. 


\section{Chapter 3}

\section{Sensitivity Analysis}

Sensitivity analysis was performed on parameters in the above model. Eleven parameters $\left(V_{9}, p_{I}, m, v_{T}\right.$, and $v_{i}$ for $\left.i=1, \ldots, 7\right)$ played a key role in determining the position of the Hopf bifurcation curve and thus analysis was performed on these individually in two different ways; increasing and decreasing each parameter by $30 \%$ of its base value, and determining the parameter variation needed to obtain a specified change in the Hopf bifurcation curve as plotted in the $(r, \mu)$ plane, where $r$ is the ventilation-perfusion ratio, and $\mu$ is the gain.

\subsection{Increasing and Decreasing Parameters By $30 \%$}

To determine the sensitivity of each parameter in the system, a reasonable range of values for the ventilation-perfusion ratio $(r)$ was established. Typical $r$ values were estimated to be 1.44 for males and 0.72 for females $\left(\dot{Q}=5 \mathrm{~L} / \mathrm{min}, \overline{\dot{V}}_{A}=7.2 \mathrm{~L} / \mathrm{min}\right.$ for

males, $\overline{\dot{V}}_{A}=3.6 \mathrm{~L} / \mathrm{min}$ for females) [25], thus a range of 0.6 to 1.6 was chosen. Little is known regarding specific gain values so a large range was chosen. The parameter values listed in Table 6.1 were chosen to be the base case for analysis. Parameters were analyzed one at a time, where $30 \%$ of each parameter was added and subtracted from the base case providing the range. To determine the sensitivity of each parameter, the area between the positive curve and base case was calculated, along with the area between the negative curve and base case. Results can be seen in Figure 3.1 and 3.2. 
Computed areas are listed in Table 3.1 where a negative area represents a downward shift in the Hopf bifurcation curve.

Results show that the model is very sensitive to the main arteries volume $\left(v_{4}\right)$ and left heart $\left(v_{2}\right)$ where corresponding areas are 0.0036 and 0.0018 respectively. All other parameters have varying degrees of sensitivity, with inspired $\mathrm{CO}_{2}$ partial pressure $\left(p_{I}\right)$ being the least sensitive (area of $1.4048 \mathrm{e}-04$ ). A decrease in parameters $V_{9}, p_{I}, v_{3}$, and $v_{4}$ and an increase in $V_{T}, v_{1}, v_{2}, v_{5}, v_{6}, v_{7}$, and $m$ result in a downward shift of the Hopf curve.

\subsection{Constant Area}

Parameter sensitivity can also be analyzed by determining the amount each parameter must increase and/or decrease to achieve an area of $\pm 0.5 / a(\approx 0.001)$ between curves. Each curve was plotted in the $(r, \mu)$ plane where the range of $r$ is defined to be 0.6 to 1.6. Results can be seen in Table 3.2 where the value and percentage, both positive and negative, are recorded. Entries without numbers indicate that an area of 0.001 could not be established. This occurs, for example, in inspired $\mathrm{CO}_{2}$ partial pressure $\left(p_{I}\right)$. The pressure can have a minimum value of zero, so, when $p_{I}$ was set equal to zero, the area was $2.3270 \mathrm{e}-0.4$ (negative); thus, an area of 0.001 will not be reached. Therefore, the model is not sensitive to $p_{I}$, which agrees with the results shown in Table 3.1. Similarly, the systemic veins together with the pulmonary arteries and right heart $\left(v_{7}\right)$ cannot be increased enough to obtain an area of 0.001 ; thus, the model is also not sensitive to $v_{7}$. The systemic capillaries volume $\left(v_{6}\right)$ has a slightly different property; like $p_{I}, v_{6}$ cannot be negative, so while decreasing the parameter, an area of 0.001 can never be reached (when $v_{6}=0.1$ the area is $6.0722 \mathrm{e}-0.4$ ). Increasing $v_{6}$, on the other hand, results in behaviour not seen in the other monotonic parameters. The systemic capillaries parameter increases monotonically until it reaches a maximum of 6.1829e-0.4 (negative). From there, even though $v_{6}$ is still increasing, the area begins 

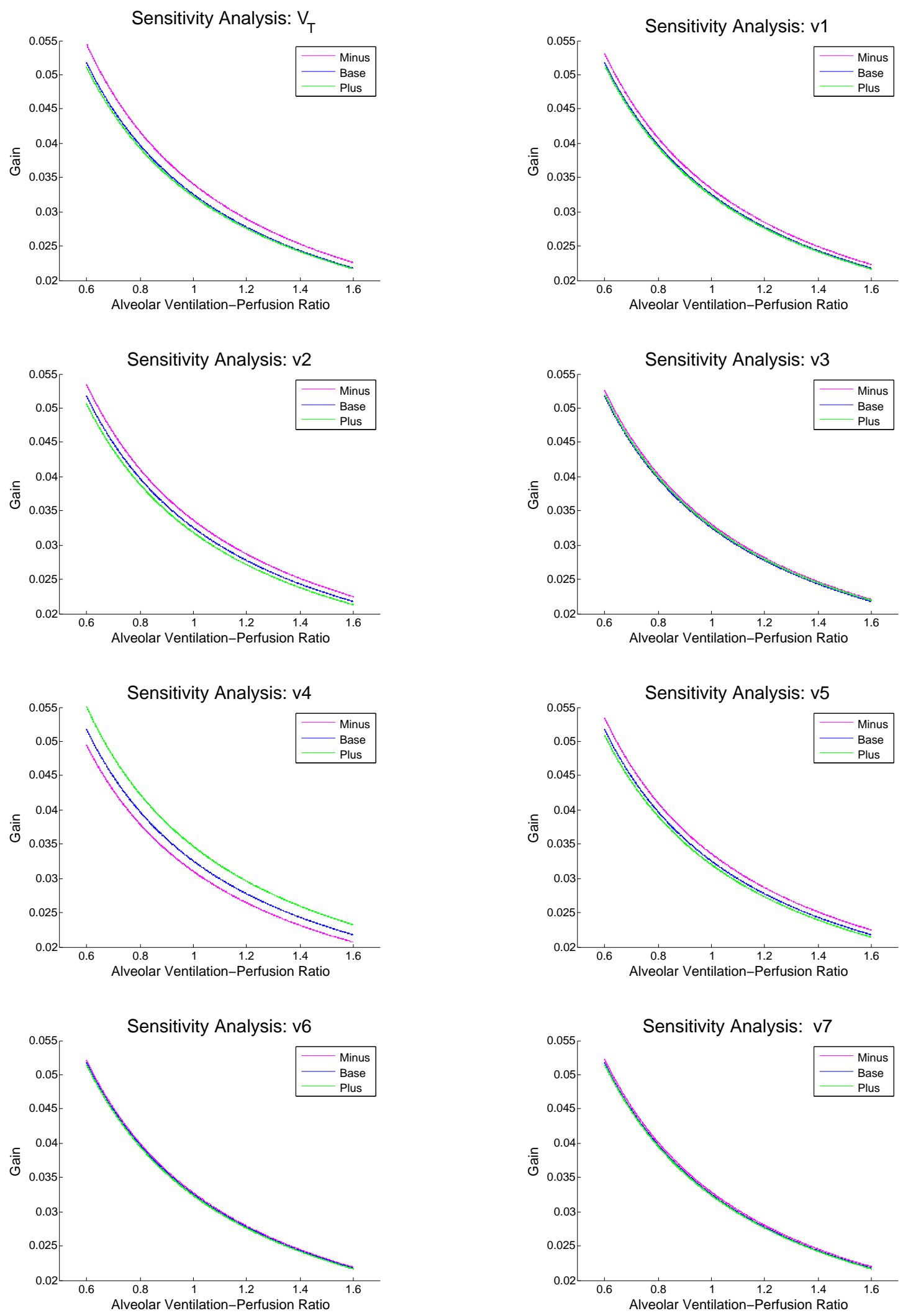

Figure 3.1: Sensitivity analysis for blood volume parameters of Dong and Langford model. 

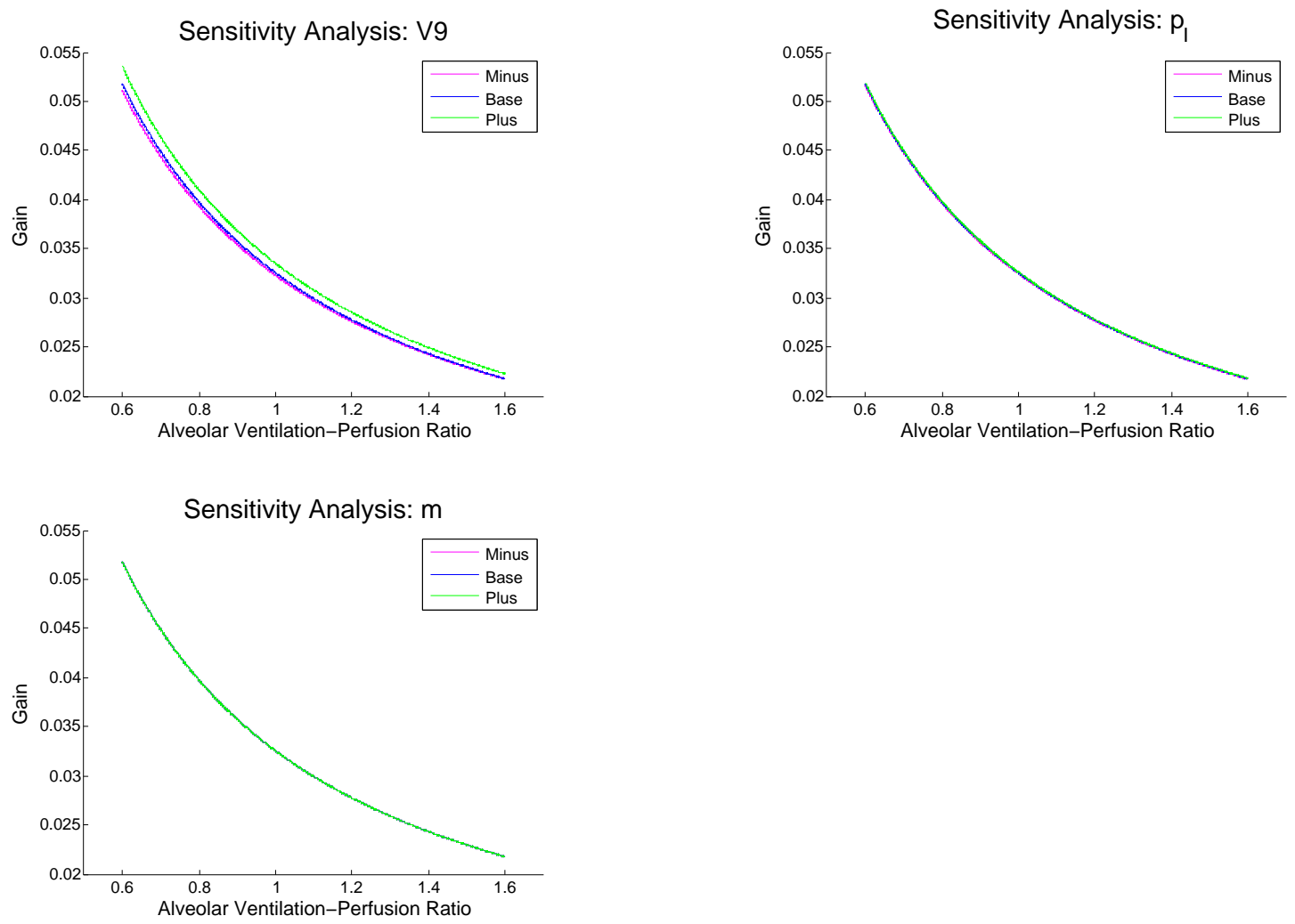

Figure 3.2: Sensitivity analysis for $V_{9}, p_{I}$, and $m$ in Dong and Langford model.

to decrease.

Both forms of sensitivity analysis conclude that the most sensitive parameters are cardiovascular efficiency $(m)$ and the main arteries $\left(v_{4}\right)$, with inspired $\mathrm{CO}_{2}$ partial pressure $\left(p_{I}\right)$ and the systemic capillaries $\left(v_{6}\right)$ being the least sensitive. 


\begin{tabular}{lcccc}
\hline Parameter & & Negative & Positive & Total \\
& & $\mathbf{3 0 \%}$ & $\mathbf{3 0 \%}$ & Area \\
\hline Alveolar gas volume & $\left(V_{9}\right)$ & $-2.5003 \mathrm{e}-04$ & $9.3810 \mathrm{e}-04$ & 0.0012 \\
Inspired $\mathrm{CO}_{2}$ partial pressure & $\left(p_{I}\right)$ & $-7.0190 \mathrm{e}-05$ & $7.0285 \mathrm{e}-05$ & $1.4048 \mathrm{e}-04$ \\
Total volume of blood in body & $\left(V_{T}\right)$ & 0.0015 & $-2.7940 \mathrm{e}-04$ & 0.0017 \\
Pulmonary veins volume & $\left(v_{1}\right)$ & $8.2294 \mathrm{e}-04$ & $-2.0128 \mathrm{e}-04$ & 0.0010 \\
Left heart volume & $\left(v_{2}\right)$ & 0.0011 & $-7.0626 \mathrm{e}-04$ & 0.0018 \\
Aorta volume & $\left(v_{3}\right)$ & $4.7221 \mathrm{e}-04$ & $2.0572 \mathrm{e}-04$ & $6.7794 \mathrm{e}-04$ \\
Main arteries volume & $\left(v_{4}\right)$ & -0.0015 & 0.0021 & 0.0036 \\
Arterioles volume & $\left(v_{5}\right)$ & 0.0010 & $-5.2003 \mathrm{e}-04$ & 0.0016 \\
Systemic capillaries volume & $\left(v_{6}\right)$ & $1.9058 \mathrm{e}-04$ & $-1.7138 \mathrm{e}-04$ & $3.6196 \mathrm{e}-04$ \\
Systemic veins, pulmonary arteries, rt & $\left(v_{7}\right)$ & $3.0466 \mathrm{e}-04$ & $-1.4398 \mathrm{e}-04$ & $4.4864 \mathrm{e}-04$ \\
heart vol. & & & & \\
Cardiovascular efficiency $\dot{M} / \dot{Q}$ & $(m)$ & 0 & 0 & 0 \\
\hline
\end{tabular}

Table 3.1: Area between two curves obtained from increasing and decreasing parameter values by $30 \%$ and subtracting from the base case.

\begin{tabular}{|l|ccc|ccc|}
\hline Base & Pos Value & Percent & Area & Neg Value & Percent & Area \\
\hline$V_{9}=3$ & 3.9500 & 32 & 0.0010 & 1.1400 & 136 & 0.0010 \\
$p_{I}=0.3$ & 1.5500 & 417 & 0.0010 & - & - & - \\
$v_{T}=5$ & 13.1000 & 162 & 0.0010 & 3.7900 & 32 & 0.0010 \\
$v_{1}=0.25$ & 0.5400 & 116 & 0.0010 & 0.1640 & 52 & 0.0010 \\
$v_{2}=0.15$ & 0.2230 & 49 & -0.0010 & 0.1070 & 40 & 0.0010 \\
$v_{3}=0.3$ & 0.5050 & 68 & 0.0010 & 0.1710 & 75 & 0.0010 \\
$v_{4}=0.5$ & 0.5760 & 15 & 0.0010 & 0.4090 & 22 & -0.0010 \\
$v_{5}=0.2$ & 0.6050 & 203 & 0.0010 & 0.1410 & 42 & 0.0010 \\
$v_{6}=0.2$ & - & - & - & - & - & - \\
$v_{7}=3.3$ & - & - & - & 1.4600 & 126 & 0.0010 \\
\hline
\end{tabular}

Table 3.2: Percentage needed to obtain an area of 0.001 both above and below the base case of the Hopf bifurcation curve. 


\section{Chapter 4}

\section{Body Mass Model}

\subsection{Introduction: Obesity}

The following section describes model adjustments applied to the model of CheyneStokes Respiration found in [15], and the corresponding results. The purpose of the adjustments was to make an accurate representation of obesity and to determine how it affects a person's likelihood of experiencing CSR. First, typical adult parameters were chosen for both males and females. Model equations were altered to account for excess mass found in obese individuals and numerical simulations were carried out.

\subsection{Model Adjustments}

\subsubsection{Body Size Adjustments}

A major factor associated with Cheyne-Stokes respiration is gender; males are more likely to experience CSR than females $[14,47]$. In order to adapt the model in $[15]$ for males and females, it was assumed that the different CSR responses of males and females are due to differences in body size, specifically mass. Frequently in the literature, a range of 'average' mass values for males and females are provided, however for this thesis, $78 \mathrm{~kg}$ and $61 \mathrm{~kg}$ are chosen to be the ideal mass for males and females respectively [17]. Non-obese individuals contain anywhere from $75 \mathrm{~mL}$ to $86 \mathrm{~mL}$ of blood per $\mathrm{kg}[3,5,46]$. Throughout this thesis, a value of $75 \mathrm{~mL} / \mathrm{kg}$ of 
blood will be used. Therefore, males and females contain approximately $5.85 \mathrm{~L}$ and $4.58 \mathrm{~L}$ of blood on average. Base parameters found in [15] were scaled according to these volumes and thus differentiation between males and females was established.

\subsubsection{Obesity Adjustments}

In order to determine if obesity plays a role in the onset of CSR, multiple adjustments must be made. It is known that the total blood volume in obese individuals increases on a volume/mass basis and

$$
\text { ideal mass }\left(\frac{m L}{k g}\right) \times(20-30) \%=\text { excess mass }\left(\frac{m L}{k g}\right)
$$

$[3,4]$. This means that we must account for the excess blood found in fat tissues [4]. Non-obese individuals contain anywhere from $75 \mathrm{~mL}$ to $86 \mathrm{~mL}$ of blood per $\mathrm{kg}$ $[3,5,46]$ and obese individuals contain $50 \mathrm{~mL}$ of blood per $\mathrm{kg}[3]$. As stated above, throughout this thesis, non-obese individuals contain $75 \mathrm{~mL}$ of blood per $\mathrm{kg}$ and for every kilogram of excess mass, the blood volume will consequently increase by $25 \mathrm{~mL} / \mathrm{kg}$ (if an individual is approximately twice the ideal mass). The resulting percentage from substituting these values into Equation 4.1 is $\approx 33 \%$. Thus, the values are a good fit; ideal mass contains $75 \mathrm{~mL} / \mathrm{kg}$ of blood and excess mass contains $25 \mathrm{~mL} / \mathrm{kg}$ of blood.

'Typical' body mass for both males and females have a wide range, however for the remainder of this thesis $78 \mathrm{~kg}$ and $61 \mathrm{~kg}$ will represent typical body mass for males and females respectively [17]. Body mass above these values was assumed to be excess, contributing to excess blood volume. The total blood volume was then determined by adding the excess to ideal blood volume.

To add the excess mass to model parameters, it was necessary to determine what

compartments experience changes in blood volume. Most excess blood seen in obese 
individuals is associated with the systemic capillaries and veins which service the fat tissues [4]. Thus, excess blood is seen in the systemic capillaries $\left(v_{6}\right)$ and the systemic veins, pulmonary arteries, and right heart $\left(v_{7}\right)$. Division of $v_{7}$ is necessary to isolate the systemic veins. It is known that $v_{7}$ typically contains $3.3 \mathrm{~L}$ of blood (see Appendix A), with the right heart and pulmonary arteries containing $0.2 \mathrm{~L}$ and $0.1 \mathrm{~L}$ respectively. The resulting systemic veins volume is $3 \mathrm{~L}$ [15]. Assume that the systemic veins and capillaries increase proportionally based on their volumes. Typical parameter values show that $v_{6}$ contains $0.2 \mathrm{~L}$ and $v_{7}$ contains $3 \mathrm{~L}$ which proportionally contribute to excess mass. Thus, $0.2 / 3.2$ of the excess blood will be placed in compartment 6 and 3.0/3.2 of the excess blood will be placed in compartment 7 . The following are added to the model where $e b$ is the excess blood.

$$
\begin{aligned}
& v_{6_{\text {obese }}}=v_{6}+e b\left(\frac{0.2}{3.2}\right), \\
& v_{7_{\text {obese }}}=v_{7}+e b\left(\frac{3.0}{3.2}\right), \\
& v_{T_{\text {obese }}}=v_{T}+e b .
\end{aligned}
$$

Calculations of excess blood volumes $(e b)$ were done by taking the amount of excess mass of the obese individual and multiply by $25 \mathrm{~mL} / \mathrm{kg}$.

In order to get an idea of how the system will behave, one can refer back to the sensitivity analysis seen in Chapter 3. For the obesity adjustments, major changes occur in $v_{6}, v_{7}$, and $v_{T}$. By referring to Figures 3.1 and 3.2 and Tables 3.1 and 3.2 it is clear that $v_{6}$ and $v_{7}$ are not sensitive at all, and $v_{T}$ is only slightly sensitive. Therefore, we should not expect to see much change to resulting Hopf bifurcation curves as body mass, and thus blood volumes increase. 


\subsection{Results}

\subsubsection{Body Size}

Scaling all blood volume parameters accordingly, corresponding male and female Hopf bifurcation curves can be seen in Figure 4.1. In all figures, the region below the Hopf curve represents a stable steady breathing pattern and the region above the curve represents the oscillatory breathing of Cheyne-Stokes Respiration. The asterisk displayed beneath the curve represents gain and ventilation-perfusion ratio values of a typical adult. The ventilation-perfusion ratio is known to have a range of $0.3-2.1$ [43]. Thus we set the $x$-axis as the range of values. Let the ventilation-perfusion ratio equal 1 and let 0.025 be a typical gain value found for both males and females in order to compare shifts in the Hopf curve [15]. Therefore, for both males and females, the asterisk rests at $(r, \mu)=(1,0.025)$.

Careful examination of Figure 4.1 shows that adult males are more likely to experience CSR because their typical parameter value lies closer to the Hopf curve than females do. When zoomed in, Figure 4.1 (c) and (d), the point where the curve and the $x$-axis intersect is closer to the the typical value for males. For males, at $r=1$, the corresponding $\mu$ value on the Hopf curve is $\mu=0.0323$, while for females at the same ventilation perfusion ratio value $\mu=0.0328$. Typical values are closer to the male curve than the female curve. This result coincides with biological literature; males are more likely to develop CSR [14].

\subsubsection{Obesity}

Multiple levels of obesity were investigated by producing curves for varying amounts of excess mass. Figure 4.2 shows Hopf curves for individuals with mass from $100 \%$ to $200 \%$ of ideal mass, in $20 \%$ increments. Results show that males and females display the same behaviour; as the amount of excess mass increases, the curve shows 
(a)

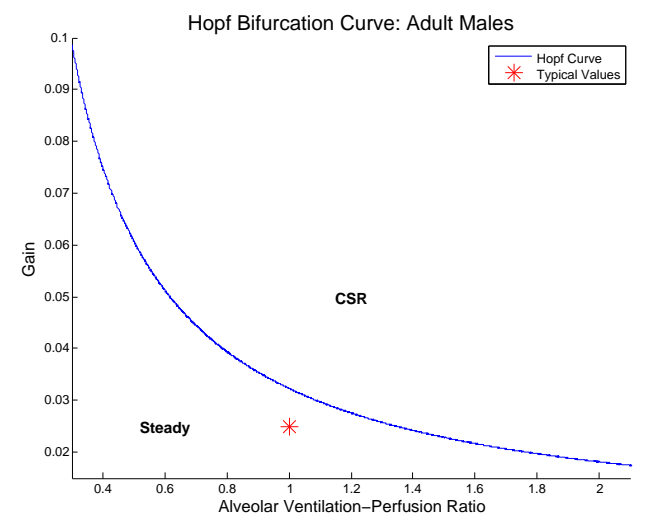

(c)

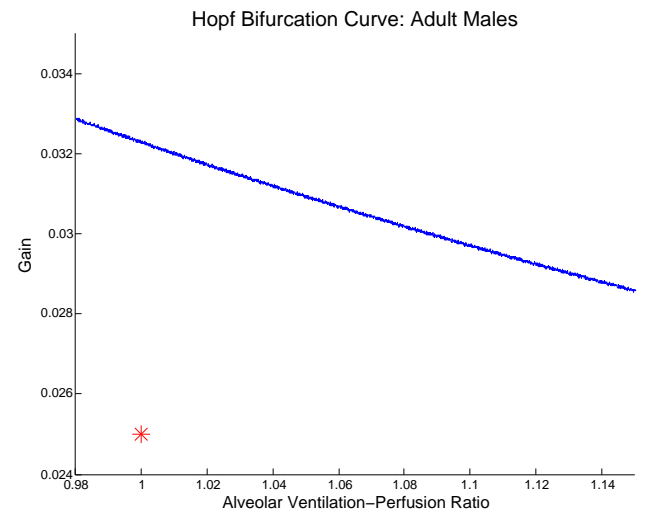

(b)

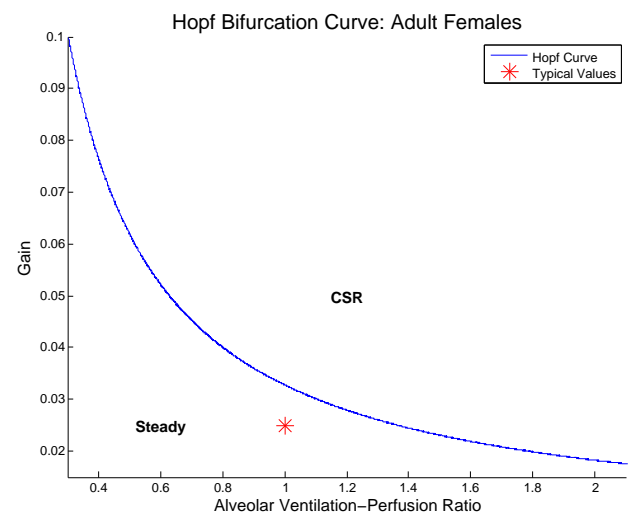

(d)

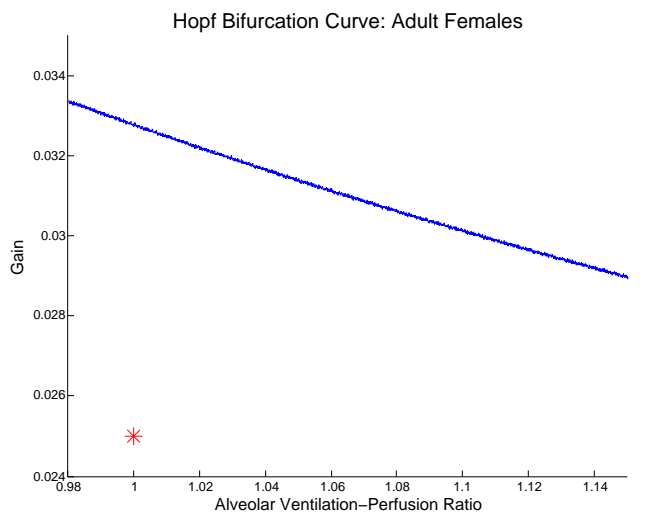

Figure 4.1: Hopf bifurcation curves for typical adults: (a) male, $v_{T}=5.32 \mathrm{~L}$, (b) female, $v_{T}=4.57 \mathrm{~L}, \quad$ (c) male zoomed in, (d) female zoomed in. Asterisks mark typical gain and alveolar ventilation-perfusion ratio values. 
a very narrow non-linear decrease. Each of these decreases in the Hopf curve is almost indistinguishable, however it represents an increase in the likelihood of an individual experiencing Cheyne-Stokes Respiration (decrease in the stable steady region).

Figure 4.2 (b) and (d) show a downward shift in the curve when the amount of excess mass increases. Although there is a decrease, it is so small that it is in essence inconsequential. We cannot make any direct conclusion regarding obesity and CSR. Increasing an individual's body mass to the extreme (excess mass $=200 \%$ of ideal mass) has no direct effect on the likelihood of developing CSR. Therefore this model does not show a significant relationship between obesity and CSR. This result is in line with our predicted outcome based on sensitivity analysis and is supported by [14], which states that obesity does not directly increase an individual's chance of developing CSR.

\subsection{Conclusion}

Altering the model produced in [15] resulted in only slight changes regarding obesity. The differences between male and female curves are very minimal. Effects of obesity alone on increasing the likelihood of CSR were not significant, however results did support biological evidence. In some subjects, both OSA and CSR can exist, but the model suggests that obesity alone is not a major risk factor for CSR [14]. 
(a)

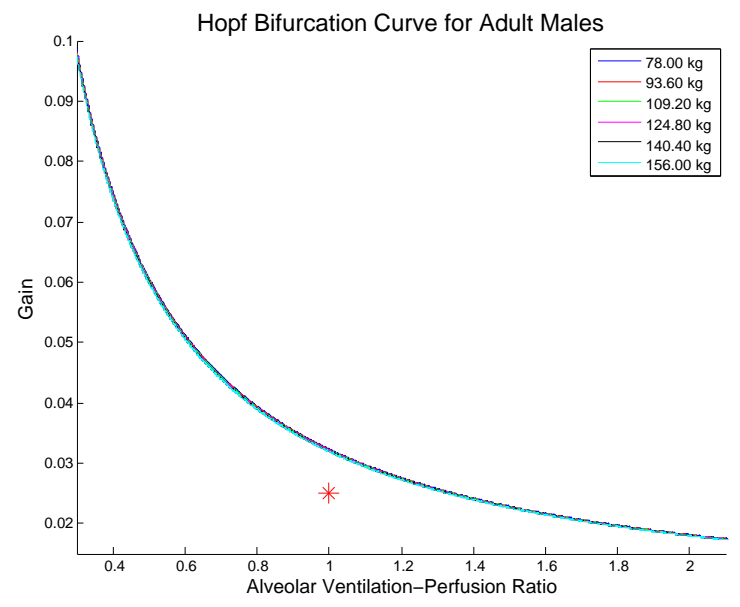

(c)

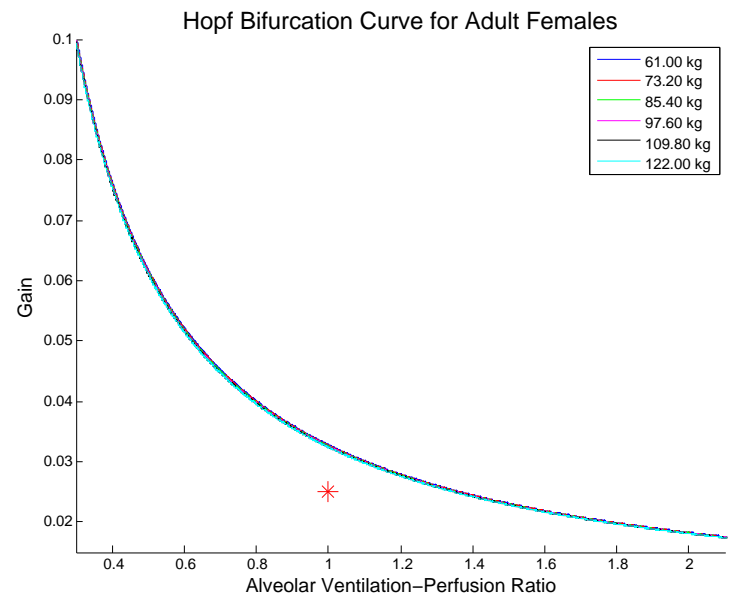

(b)

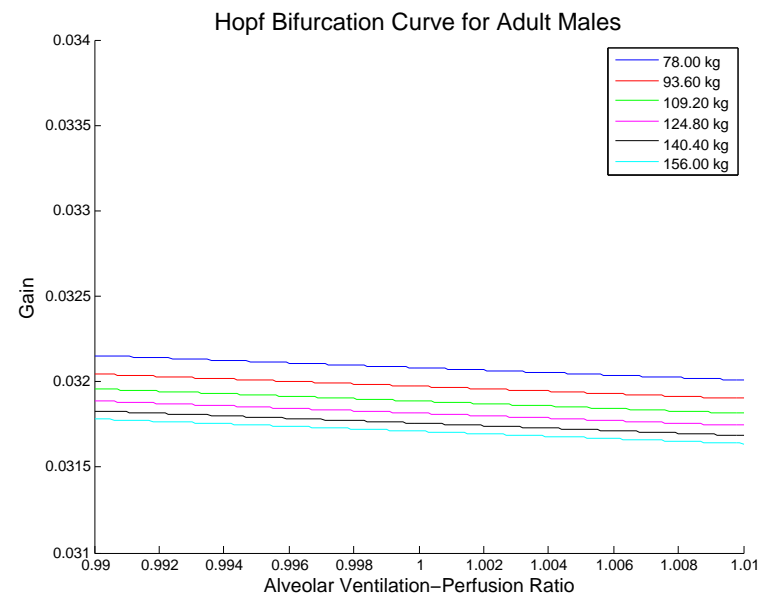

(d)

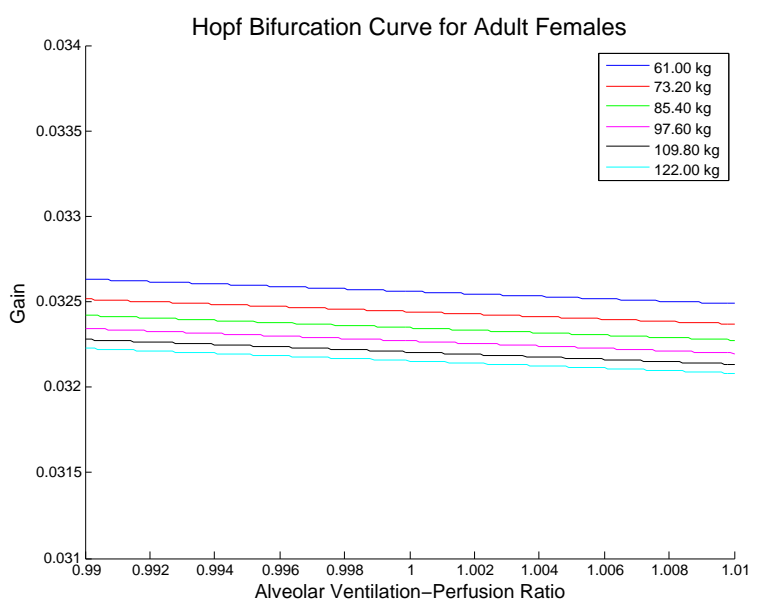

Figure 4.2: Hopf bifurcation curves: (a) obese male, (b) obese male zoomed in, (c) obese female, (d) obese female zoomed in. Asterisks denote gain and alveolar ventilation-perfusion ratio values for typical subjects. 


\section{Chapter 5}

\section{Fluid Shift Model}

\subsection{Introduction: Fluid Shift}

Recent medical studies suggest that a major component in the onset of Cheyne-Stokes Respiration is fluid shift in the body, upon entering the recumbent position $[38,40]$. As the fluid shifts, a considerable amount comes to rest in the neck, where the added fluid appears to contribute to the onset of CSR [40]. Below, a mathematical model is constructed which aims to shed some light on how the likelihood of developing CSR changes due to body position.

Despite the fact the there are a large number of mathematical models relating to the cardio-respiratory system, few have considered the idea of fluid shift. Those that do base it on orthostatic stress $[16,18,24]$. Orthostatic stress occurs when a person is in the upright position; gravity forces blood to pool in the lower body [18]. Shifts in fluids are measured while standing, which can be extended to our model, however the recumbent position is not considered [18, 24].

It is clear that there are a large number of mathematical models relating to CSR, and a few that go further to investigate fluid shifts seen in orthostatic stress. None however have tackled the question of fluid shift upon entering the recumbent position. The purpose of this thesis is to construct a mathematical model of the cardiovascular

and respiratory systems that can be used to investigate the likelihood of obtaining CSR due to body position. 


\subsection{Mathematical Model}

For the purpose of modelling CSR, we divide the human body into the circulatory system, respiratory system, and the feedback control system, as in [6, 15]. Below, each system is described and a model of the flow of and response to carbon dioxide concentrations found in the blood is produced.

\subsubsection{Circulatory System}

The circulatory system is divided into 15 compartments in order to model the flow of blood. These compartments are: (1) Pulmonary Veins, (2) Left Heart, (3) Aorta, (4) Carotid Artery, (5) Main Arteries, (6) Arterioles in the Neck, (7) Arterioles in the Abdomen, (8) Arterioles in the Legs, (9) Systemic Capillaries in the Neck, (10) Systemic Capillaries in the Abdomen, (11) Systemic Capillaries in the Legs, (12) Jugular Vein, (13) Systemic Veins, (14) Right Heart and Pulmonary Arteries, and (15) Pulmonary Capillaries. Figure 5.1 shows the location of each compartment in the heart and circulatory system.

Beginning in the pulmonary veins, blood travels through the left heart and into the aorta. From there, the system branches into the main arteries and the upper loop which consists of the head, neck and arms. Traveling into the main arteries presents a second branching point where blood either travels to the middle loop which includes the abdomen or the lower loop containing the legs. After a complete cycle, all three loops merge in the right heart. Here blood travels to the pulmonary arteries, pulmonary capillaries and back into the pulmonary veins where the cycle begins again. As in [15], we assume the pulmonary arteries contain such a small volume that it is appropriate to combine it with the right heart in compartment 14 .

To mathematically account for the branching into the upper, middle and lower loops seen in Figure 5.1 upon leaving the aorta, and again leaving the main arteries,

it is necessary to break down the blood flow rate at rest, $\dot{Q}$, into three parts. Let $J$ 


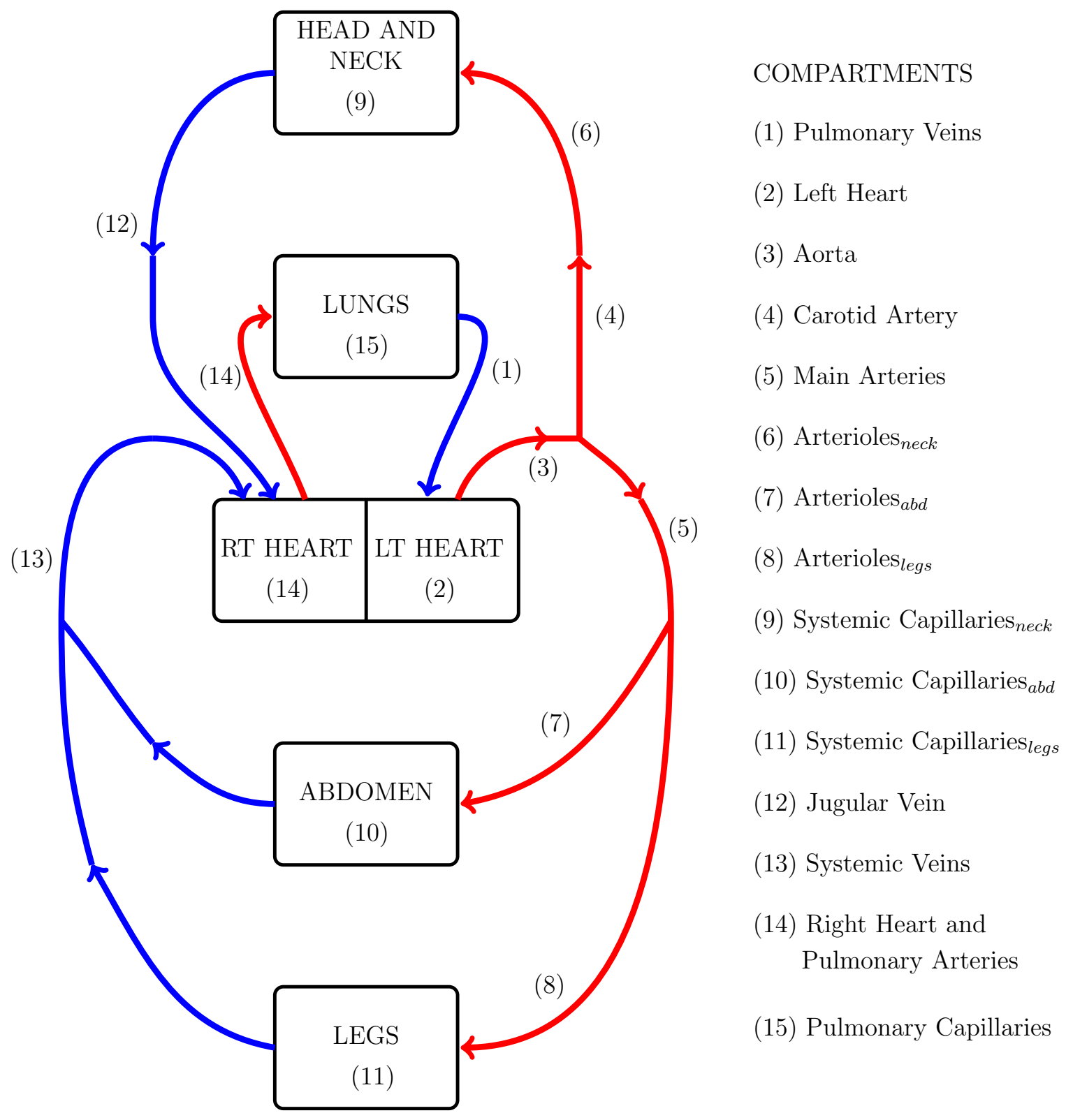

Figure 5.1: Heart and Circulatory System. Red lines indicate arteries, containing highly oxygenated blood. Blue lines represent veins, carrying blood back to the lungs to become re-oxygenated. 
denote the fraction of $\dot{Q}$ contributing to the specific branch taken, where subscripts $A, L$, and $N$ represent the abdomen, legs and neck respectively. Then, $J_{A}, J_{L}$, and $J_{N}$ are the fractions of the blood flow entering the abdomen, legs, and neck where

$$
J_{A}+J_{L}+J_{N}=1
$$

Similarly, let $\dot{M}$ be the total rate at which $\mathrm{CO}_{2}$ is produced by metabolism at rest. Let $K_{A}, K_{L}$, and $K_{N}$ represent the fraction of $\dot{M}$ that occurs in the abdomen, legs, and neck, then

$$
K_{A}+K_{L}+K_{N}=1
$$

Except for the capillary compartments $(9,10,11$, and 15) it is assumed that the $\mathrm{CO}_{2}$ concentration does not change as it passes through each of the other compartments [15]. Let $c_{i}$ be the concentration of $\mathrm{CO}_{2}$, where $i$ denotes the compartment. Then, let $v_{i}$ represent the volume of blood in compartment $i$. The equations corresponding to the compartments before branching are of the form

$$
\frac{d\left(v_{i} c_{i}\right)}{d t}=\dot{Q} c_{i-1}-\dot{Q} c_{i}, \quad i=1,2,3
$$

where $c_{0}=c_{15}$.

Compartment 14 combines the $\mathrm{CO}_{2}$ concentrations in lower, middle and upper loops in the right heart and pulmonary arteries. The relation is given by

$$
\frac{d\left(v_{14} c_{14}\right)}{d t}=\left(J_{L}+J_{A}\right) \dot{Q} c_{13}+J_{N} \dot{Q} c_{12}-\dot{Q} c_{14}
$$

where the fraction of the blood flow in the middle and lower loop was added to the fraction of the blood flow in the upper loop to produce the total blood flow rate in the right heart and pulmonary arteries.

Metabolic processes throughout the body play a large role in altering the amount 
of $\mathrm{CO}_{2}$ present in specific compartments. Major changes in $\mathrm{CO}_{2}$ occur in exchange vessels found in the lungs, capillaries, and small veins [1]. In the lungs the blood gains $\mathrm{O}_{2}$ while losing $\mathrm{CO}_{2}$ whereas in the systemic capillaries and veins $\mathrm{O}_{2}$ is lost and $\mathrm{CO}_{2}$ is gained $[1,15]$. Therefore in this model, $\mathrm{CO}_{2}$ is added in compartments 9,10 , and 11, while it is removed in compartment 15 .

For compartment 15, the pulmonary capillaries, the amount of $\mathrm{CO}_{2}$ is governed by

$$
\frac{d\left(v_{15} c_{15}\right)}{d t}=\dot{Q} c_{14}-\dot{Q} c_{15}-\dot{R}
$$

where $\dot{R}$ is the removal rate of $\mathrm{CO}_{2}$ from the pulmonary capillaries to the alveoli of the lungs.

The following sections describe how $\mathrm{CO}_{2}$ in the blood is transferred through each of the compartments in the upper, middle and lower loops following the branching of the aorta and main arteries until they reconnect again in the right heart.

\section{Upper Body Circulation}

The upper loop begins in the aorta where a fraction of the blood flow, $J_{N}$, begins to travel up the carotid artery into the arterioles, followed by the systemic capillaries of the head, neck and arms. In the capillaries, $\mathrm{CO}_{2}$ produced by metabolism is added to the current $\mathrm{CO}_{2}$ concentration. From there $\mathrm{CO}_{2}$ enriched blood travels down the jugular vein into the right heart where it meets the flow from the middle and lower loop. 
The model equations corresponding to the upper loop are

$$
\begin{aligned}
\frac{d\left(v_{4} c_{4}\right)}{d t} & =J_{N}\left(\dot{Q} c_{3}-\dot{Q} c_{4}\right) \\
\frac{d\left(v_{6} c_{6}\right)}{d t} & =J_{N}\left(\dot{Q} c_{4}-\dot{Q} c_{6}\right) \\
\frac{d\left(v_{9} c_{9}\right)}{d t} & =J_{N}\left(\dot{Q} c_{6}-\dot{Q} c_{9}\right)+K_{N} \dot{M} \\
\frac{d\left(v_{12} c_{12}\right)}{d t} & =J_{N}\left(\dot{Q} c_{9}-\dot{Q} c_{12}\right) .
\end{aligned}
$$

\section{Middle Body Circulation}

The middle loop also begins in the aorta, where $\left(J_{A}+J_{L}\right)$ of the blood flow continues to the main arteries. Here it reaches a second branching point. A fraction $J_{A}$ of the blood flow in the main arteries gets sent to the middle loop, containing the abdomen, where blood continues to flow into the abdominal systemic capillaries. Here, $\mathrm{CO}_{2}$ is produced by metabolism and is added to the current concentration. From the capillaries it travels out into the systemic veins where it connects with the lower loop.

The middle loop model equations are:

$$
\begin{aligned}
\frac{d\left(v_{5} c_{5}\right)}{d t} & =\left(J_{A}+J_{L}\right)\left(\dot{Q} c_{3}-\dot{Q} c_{5}\right) \\
\frac{d\left(v_{7} c_{7}\right)}{d t} & =J_{A}\left(\dot{Q} c_{5}-\dot{Q} c_{7}\right) \\
\frac{d\left(v_{10} c_{10}\right)}{d t} & =J_{A}\left(\dot{Q} c_{7}-\dot{Q} c_{10}\right)+K_{A} \dot{M} .
\end{aligned}
$$

\section{Lower Body Circulation}

The lower loop consists of the legs and is similar to the middle loop. A fraction $J_{L}$ of the blood found in the main arteries enters the arterioles of the legs and continues into the systemic capillaries. Again metabolism produces $\mathrm{CO}_{2}$, adding to the concentration already present. From the capillaries of the legs blood flows to the systemic veins where it meets the blood from the systemic capillaries of the abdomen, and together travel up to the right heart. Once there, all three loops continue to circulate 


\begin{tabular}{|c|c|c|c|}
\hline Region & Compartment & Region & Compartment \\
\hline Neck & $\begin{array}{l}\text { Carotid Artery } \\
\text { Arterioles }_{N} \\
\text { Systemic Capillaries }_{N} \\
\text { Jugular Vein }\end{array}$ & Abdomen & $\begin{array}{l}\text { Arterioles }_{A} \\
\text { Systemic Capillaries } \\
\text { Systemic Veins } \\
\text { Sy }\end{array}$ \\
\hline Thorax & $\begin{array}{l}\text { Right Heart } \\
\text { Pulmonary Arteries } \\
\text { Pulmonary Capillaries } \\
\text { Pulmonary Veins } \\
\text { Left Heart } \\
\text { Aorta } \\
\text { Main Arteries }\end{array}$ & Legs & $\begin{array}{l}\text { Arterioles }_{L} \\
\text { Systemic Capillaries } \\
\text { Systemic Veins } \\
\text { Sy }\end{array}$ \\
\hline
\end{tabular}

Table 5.1: Classification of compartments into body regions.

as one.

The model equations for the circulation of the lower body are as follows:

$$
\begin{aligned}
\frac{d\left(v_{8} c_{8}\right)}{d t} & =J_{L}\left(\dot{Q} c_{5}-\dot{Q} c_{8}\right) \\
\frac{d\left(v_{11} c_{11}\right)}{d t} & =J_{L}\left(\dot{Q} c_{8}-\dot{Q} c_{11}\right)+K_{L} \dot{M} \\
\frac{d\left(v_{13} c_{13}\right)}{d t} & =J_{L} \dot{Q} c_{11}+J_{A} \dot{Q} c_{10}-\left(J_{L}+J_{A}\right) \dot{Q} c_{13} .
\end{aligned}
$$

\section{Fluid Shift Body Regions}

Fluid shift is typically measured in four regions of the body; the neck, thorax, abdomen and legs. In order to model the fluid shift, each compartment of the model must be classified into one of these 4 regions. Refer to Table 5.1 for classifications.

\subsubsection{Respiratory System}

The respiratory system assumptions and model equations are as in [15]. Below is a brief overview of the model equations.

The respiratory system provides an additional compartment to the model, the alveoli of the lungs (compartment 16). Here, $\mathrm{CO}_{2}$ is in a gaseous form and diffuses 
into the pulmonary capillaries through the respiratory membrane.

Let $p_{i}$ be the partial pressure in compartment $i$. The empirical linear relationship between the concentration and partial pressure of $\mathrm{CO}_{2}$ is $[6,15]$

$$
p_{i}=\frac{c_{i}}{8}-20
$$

It is reasonable to assume that the partial pressures in the capillaries and alveoli are equal $[22,25,45]$

$$
p_{16}=p_{15}=\left[\frac{c_{15}}{8}-20\right]
$$

indicating that once $p_{16}$ is known, $c_{15}$ is also known.

$\mathrm{CO}_{2}$ is determined by the partial pressure in the lungs so changes are modelled in the partial pressure instead of concentration. The transfer of $\mathrm{CO}_{2}$ into and out of the lungs is intrinsically instantaneous $[15,22,25]$. Letting $\dot{V}_{A}$ be the alveolar ventilation rate, $V_{G}$ the alveolar gas volume, and $p_{I}$ the inspired $\mathrm{CO}_{2}$ partial pressure the relation is

$$
\frac{d p_{16}}{d t}=d \cdot \dot{Q}\left(c_{14}-c_{15}\right)-\frac{\dot{V}_{A}}{V_{G}}\left(p_{16}-p_{I}\right) .
$$

Here, $d$ is called the perfusion constant (see Appendix 6.1). Combining equations (5.8) and (5.9) to eliminate $p_{16}$ results in

$$
\frac{d\left(c_{15}\right)}{d t}=8 d \cdot \dot{Q}\left(c_{14}-c_{15}\right)-\frac{\dot{V}_{A}}{V_{G}}\left(c_{15}-160-8 p_{I}\right) .
$$

\subsubsection{Control System}

A full description of the feedback control system is presented in [15]. Below is a brief overview of the model equations.

The feedback control system governs the concentration of $\mathrm{CO}_{2}$ in the blood. It is monitored by two types of chemoreceptors in the brain; central and peripheral. The peripheral chemoreceptors play a major role in periodic breathing while the central 


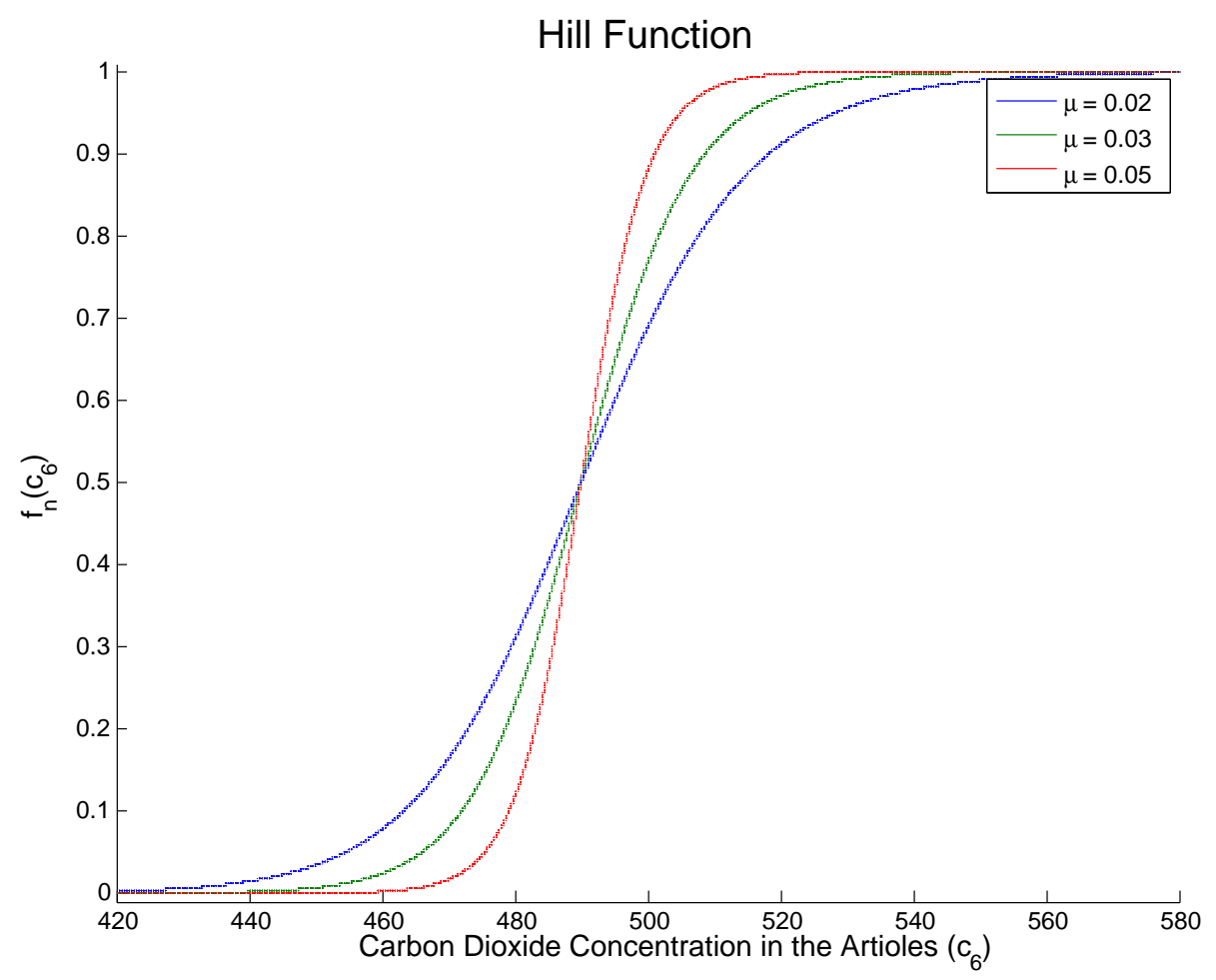

Figure 5.2: Hill functions with $a=490$. Typical $\mu$ value is 0.03 corresponding to $n=59$.

are not involved $[15,42]$. Thus, our model contains only peripheral chemoreceptors whose response is modelled using a Hill function

$$
f_{n}\left(c_{6}\right)=\frac{c_{6}^{n}}{a^{n}+c_{6}^{n}}
$$

Here $c_{6}$ is the concentration of $\mathrm{CO}_{2}$ in compartment 6 which is where the chemoreceptors detect changes in $\mathrm{CO}_{2}$ levels $[27,35], a$ is a constant and $n$ determines the slope of the curve. See Figure 5.2.

The slope or gain $(\mu)$ of the function at $c_{6}=a$ characterizes the sensitivity of the feedback control, and is determined by

$$
\mu=\frac{n}{4 a}
$$

Using the Hill function to model the response of the chemoreceptors to changes in 
$\mathrm{CO}_{2}$ concentration, and the assumption that the breathing rate and response depend purely on the chemoreceptors, the feedback control system is

$$
\dot{V}_{A}=\overline{\dot{V}}_{A} \cdot \frac{2 \cdot c_{6}^{n}}{a^{n}+c_{6}^{n}}
$$

That is, the ventilation rate is equal to two times the midpoint ventilation rate, $\overline{\dot{V}}_{A}$, multiplied by the Hill function. Thus, at the concentration midpoint, when $\left(a=c_{6}\right)$, the ventilation rate is equal to the midpoint ventilation rate.

Substituting the equation for the control system (5.13) into the respiratory equation (5.10) produces the final model equation

$$
\frac{d\left(c_{15}\right)}{d t}=8 d \dot{Q}\left(c_{14}-c_{15}\right)-\frac{\overline{\dot{V}}_{A}}{V_{G}}\left[\frac{2 \cdot c_{6}^{n}}{a^{n}+c_{6}^{n}}\right]\left(c_{15}-160-8 p_{I}\right) .
$$

\subsubsection{Model Equations}

Combining the equations previously derived, the dynamical system modelling CheyneStokes Respiration in this study is 


$$
\begin{aligned}
\frac{d\left(v_{1} c_{1}\right)}{d t} & =\dot{Q} c_{15}-\dot{Q} c_{1} \\
\frac{d\left(v_{2} c_{2}\right)}{d t} & =\dot{Q} c_{1}-\dot{Q} c_{2} \\
\frac{d\left(v_{3} c_{3}\right)}{d t} & =\dot{Q} c_{2}-\dot{Q} c_{3} \\
\frac{d\left(v_{4} c_{4}\right)}{d t} & =J_{N}\left(\dot{Q} c_{3}-\dot{Q} c_{4}\right) \\
\frac{d\left(v_{5} c_{5}\right)}{d t} & =\left(J_{A}+J_{L}\right)\left(\dot{Q} c_{3}-\dot{Q} c_{5}\right) \\
\frac{d\left(v_{6} c_{6}\right)}{d t} & =J_{N}\left(\dot{Q} c_{4}-\dot{Q} c_{6}\right) \\
\frac{d\left(v_{7} c_{7}\right)}{d t} & =J_{A}\left(\dot{Q} c_{5}-\dot{Q} c_{7}\right) \\
\frac{d\left(v_{8} c_{8}\right)}{d t} & =J_{L}\left(\dot{Q} c_{5}-\dot{Q} c_{8}\right) \\
\frac{d\left(v_{9} c_{9}\right)}{d t} & =J_{N}\left(\dot{Q} c_{6}-\dot{Q} c_{9}\right)+K_{N} \dot{M} \\
\frac{d\left(v_{10} c_{10}\right)}{d t} & =J_{A}\left(\dot{Q} c_{7}-\dot{Q} c_{10}\right)+K_{A} \dot{M} \\
\frac{d\left(v_{11} c_{11}\right)}{d t} & =J_{L}\left(\dot{Q} c_{8}-\dot{Q} c_{11}\right)+K_{L} \dot{M} \\
\frac{d\left(v_{12} c_{12}\right)}{d t} & =J_{N}\left(\dot{Q} c_{9}-\dot{Q} c_{12}\right) \\
\frac{d\left(v_{13} c_{13}\right)}{d t} & =J_{L} \dot{Q} c_{11}+J_{A} \dot{Q} c_{10}-\left(J_{L}+J_{A}\right) \dot{Q} c_{13} \\
\frac{d\left(v_{14} c_{14}\right)}{d t} & =\left(J_{L}+J_{A}\right) \dot{Q} c_{13}+J_{N} \dot{Q} c_{12}-\dot{Q} c_{14} \\
\frac{d\left(c_{15}\right)}{d t} & =8 d \dot{Q}\left(c_{14}-c_{15}\right)-\frac{\dot{V}_{A}}{V_{G}}\left[\frac{2 \cdot c_{6}^{n}}{a^{n}+c_{6}^{n}}\right]\left(c_{15}-160-8 p_{I}\right) \\
& =1
\end{aligned}
$$

where $J_{A}+J_{L}+J_{N}=1 \quad$ and $\quad K_{A}+K_{L}+K_{N}=1$.

\subsection{Nondimensionalized Variables}

To change to dimensionless variables, similar adjustments were performed as in [15]. Simplification of the model equations is done by letting $c_{i}=y_{i} a$, and multiplying each equation by $v_{T} / \dot{Q}$, where $v_{T}$ is the total blood volume. The following dimensionless 
variables are also defined, where $i=1, \ldots, 15$ :

$$
\begin{gathered}
s=t \cdot\left[\dot{Q} / v_{T}\right], \quad \hat{d}=8 d v_{T}, \quad m=\frac{\dot{M}}{\dot{Q}}, \\
r=\frac{\overline{\dot{V}}_{A}}{\dot{Q}}, \quad \hat{v}_{i}=\frac{v_{i}}{v_{T}}, \quad \hat{V}_{G}=\frac{V_{G}}{v_{T}} .
\end{gathered}
$$

Applying these changes produces the following dimensionless model:

$$
\begin{aligned}
\frac{d y_{1}}{d s} & =\frac{1}{\hat{v}_{1}}\left(y_{15}-y_{1}\right) \\
\frac{d y_{2}}{d s} & =\frac{1}{\hat{v}_{2}}\left(y_{1}-y_{2}\right) \\
\frac{d y_{3}}{d s} & =\frac{1}{\hat{v}_{3}}\left(y_{2}-y_{3}\right) \\
\frac{d y_{4}}{d s} & =\frac{J_{N}}{\hat{v}_{4}}\left(y_{3}-y_{4}\right) \\
\frac{d y_{5}}{d s} & =\frac{\left(J_{A}+J_{L}\right)}{\hat{v}_{5}}\left(y_{3}-y_{5}\right) \\
\frac{d y_{6}}{d s} & =\frac{J_{N}}{\hat{v}_{6}}\left(y_{4}-y_{6}\right) \\
\frac{d y_{7}}{d s} & =\frac{J_{A}}{\hat{v}_{7}}\left(y_{5}-y_{7}\right) \\
\frac{d y_{8}}{d s} & =\frac{J_{A}}{\hat{v}_{8}}\left(y_{5}-y_{8}\right) \\
\frac{d y_{9}}{d s} & =\frac{1}{\hat{v}_{9}}\left(J_{N}\left(y_{6}-y_{9}\right)+\frac{m K_{N}}{a}\right) \\
\frac{d y_{10}}{d s} & =\frac{1}{\hat{v}_{10}}\left(J_{A}\left(y_{7}-y_{10}\right)+\frac{m K_{A}}{a}\right) \\
\frac{d y_{11}}{d s} & =\frac{1}{\hat{v}_{11}}\left(J_{L}\left(y_{8}-y_{11}\right)+\frac{m K_{L}}{a}\right) \\
\frac{d y_{12}}{d s} & =\frac{J_{N}}{\hat{v}_{12}}\left(y_{9}-y_{12}\right) \\
\frac{d y_{13}}{d s} & =\frac{1}{\hat{v}_{13}}\left(J_{L} y_{11}+J_{A} y_{10}-\left(J_{L}+J_{A}\right) y_{13}\right) \\
\frac{d s}{d s} & =\frac{1}{\hat{v}_{14}}\left(\left(J_{L}+J_{A}\right) y_{13}+J_{N} y_{12}-y_{14}\right) \\
\left.y_{15}\right)-\frac{2 r}{\hat{V}_{G}} & 1+y_{6}^{n} \\
\frac{1}{6}\left(y_{15}-\right. & \left.160+8 p_{I}\right) \\
&
\end{aligned}
$$


where $J_{A}+J_{L}+J_{N}=1 \quad$ and $\quad K_{A}+K_{L}+K_{N}=1$.

Equilibrium values were determined by setting each differential equation equal to zero. Let $y^{*}$ denote equilibrium solutions. Dimensionless equilibrium values are

$$
\begin{aligned}
y_{1}^{*}=y_{2}^{*}=y_{3}^{*}=y_{4}^{*}=y_{5}^{*}=y_{6}^{*}=y_{7}^{*}=y_{8}^{*} & =y_{15}^{*} \\
y_{9}^{*} & =y_{12}^{*} \\
y_{9}^{*} & =y_{1}^{*}+\left(\frac{K_{N}}{J_{N}}\right) \frac{m}{a} \\
y_{10}^{*} & =y_{1}^{*}+\left(\frac{K_{A}}{J_{A}}\right) \frac{m}{a} \\
y_{11}^{*} & =y_{1}^{*}+\left(\frac{K_{L}}{J_{L}}\right) \frac{m}{a} \\
y_{13}^{*} & =y_{1}^{*}+\left(\frac{K_{L}+K_{A}}{J_{L}+J_{A}}\right) \frac{m}{a} \\
y_{14}^{*} & =y_{1}^{*}+\frac{m}{a}
\end{aligned}
$$

where

$$
0=\frac{\hat{d} m}{a}-\frac{2 r}{\hat{V}_{G}}\left[\frac{\left[y_{1}^{*}\right]^{n}}{1+\left[y_{1}^{*}\right]^{n}}\right]\left(y_{1}^{*}-\frac{160+8 p_{I}}{a}\right)
$$

Values of $a$, the concentration of $\mathrm{CO}_{2}$ in the blood at which the chemoreceptor response is half saturated, typically range from $470 \mathrm{~mL} / \mathrm{L}$ to $510 \mathrm{~mL} / \mathrm{L}[6,15,30]$. Table 5.2 shows $y_{1}^{*}$ values resulting from substituting respective values of $a$ into Equation 5.17. Equilibrium values can now be determined.

\begin{tabular}{|c|c|}
\hline$a$ & $y_{1}^{*}$ \\
\hline $470 \mathrm{~mL} / \mathrm{L}$ & 0.9999 \\
$480 \mathrm{~mL} / \mathrm{L}$ & 0.9984 \\
$490 \mathrm{~mL} / \mathrm{L}$ & 0.9970 \\
$500 \mathrm{~mL} / \mathrm{L}$ & 0.9957 \\
$510 \mathrm{~mL} / \mathrm{L}$ & 0.9946 \\
\hline
\end{tabular}

Table 5.2: Solutions to Equation 5.17 for respective $a$ values.

The concentration of $\mathrm{CO}_{2}, a$, has been chosen to be $490 \mathrm{~mL} / \mathrm{L}$, therefore $y_{1}^{*}=$ 
0.9970. Corresponding equilibrium values for the dimensionless system are

$$
\begin{aligned}
y_{1}^{*}=y_{2}^{*}=y_{3}^{*}=y_{4}^{*}=y_{5}^{*}=y_{6}^{*}=y_{7}^{*}=y_{8}^{*}=y_{15}^{*} & =0.9970 \\
y_{9}^{*} & =y_{12}^{*} \\
y_{9}^{*} & =y_{1}^{*}+\left(\frac{K_{N}}{J_{N}}\right) \frac{m}{a} \\
y_{10}^{*} & =y_{1}^{*}+\left(\frac{K_{A}}{J_{A}}\right) \frac{m}{a} \\
y_{11}^{*} & =y_{1}^{*}+\left(\frac{K_{L}}{J_{L}}\right) \frac{m}{a} \\
y_{13}^{*} & =y_{1}^{*}+\left(\frac{K_{L}+K_{A}}{J_{L}+J_{A}}\right) \frac{m}{a} \\
y_{14}^{*} & =y_{1}^{*}+\frac{m}{a} .
\end{aligned}
$$

That is

$$
\begin{array}{r}
y_{1}^{*}=y_{2}^{*}=y_{3}^{*}=y_{4}^{*}=y_{5}^{*}=y_{6}^{*}=y_{7}^{*}=y_{8}^{*}=y_{15}^{*}=0.9970 \\
y_{9}^{*}=y_{12}^{*}=1.061 \\
y_{10}^{*}=1.096 \\
y_{11}^{*}=0.982 \\
y_{13}^{*}=1.058 \\
y_{14}^{*}=1.059 .
\end{array}
$$




\subsection{Model Analysis}

\subsubsection{Hopf Bifurcation}

Hopf bifurcation theory was used to analyze the system of differential equations that represent the cardio-respiratory system. The normalized equilibrium point of the system is given by $y^{*}$, seen above in Equation 5.18 and the Jacobian matrix is given in Table 5.4 .

Eigenvalues are shown in Table 5.3 for all seven subjects in both the standing and supine positions, where the equilibrium point is given by (5.19) and parameter values are given in Appendix B. All eigenvalues have negative real part and the equilibrium point found in equation 5.18 is asymptotically stable (this is true for all 7 subjects). Further, there are complex conjugate paris of eigenvalues. When a given pair, say

$\lambda(\mu)$ and $\bar{\lambda}(\mu)$, cross the imaginary axis in the complex eigen-plane as $\mu$ varies at a critical point, say $\mu=\mu_{0}$, limit cycles emerge [33]. Here, $\mu$ values are greater than $\mu_{0}$ and thus the system of ODEs gives rise to stable, supercritical bifurcations. 


\begin{tabular}{|c|c|c|c|c|c|}
\hline Subject & $\begin{array}{l}\text { Eigenvalues } \\
\text { Standing } \\
\end{array}$ & Supine & Subject & $\begin{array}{l}\text { Eigenvalues } \\
\text { Standing } \\
\end{array}$ & Supine \\
\hline Subject 1 & $\begin{array}{l}-23.8899 \pm 0.0012 i \\
-15.8084 \\
-15.7376 \\
-8.6068 \\
-4.4734 \pm 3.8857 i \\
-5.5392 \\
0.4915 \pm 2.1080 i \\
-2.2971 \\
-0.3576 \\
-1.2548 \pm 0.0619 i \\
-1.017\end{array}$ & $\begin{array}{l}-21.1196 \pm 0.0019 i \\
-11.6134 \\
-11.239 \\
-8.5757 \\
-4.0661 \pm 3.9299 i \\
-5.6645 \\
0.5133 \pm 2.0398 i \\
-2.3907 \\
-1.2949 \pm 0.0640 i \\
-0.3564 \\
-0.7471\end{array}$ & Subject 5 & $\begin{array}{l}-20.2346 \pm 0.0023 i \\
-13.3496 \\
-13.1442 \\
-8.7833 \\
-4.3687 \pm 4.0655 i \\
-5.4397 \\
0.5109 \pm 2.1312 i \\
-2.4658 \\
-0.3426 \\
-1.2865 \pm 0.0849 i \\
-0.8583\end{array}$ & $\begin{array}{l}-18.6070 \pm 0.0024 i \\
-11.3959 \\
-11.1072 \\
-3.8853+3.7542 i \\
-8.0873 \\
-6.4117 \\
0.5048 \pm 1.9602 i \\
-2.237 \\
-1.5045 \\
-1.1875 \\
-0.3788 \\
-0.7332\end{array}$ \\
\hline Subject 2 & $\begin{array}{l}-26.0664 \pm 0.0056 i \\
-20.3619 \\
-19.8902 \\
-14.5754 \\
-7.3032 \pm 6.3149 i \\
0.4825 \pm 3.1867 i \\
-4.5072 \\
-3.4554 \\
-2.2947 \\
-0.2469 \\
-1.3083 \\
-0.83\end{array}$ & $\begin{array}{l}-27.0366 \pm 0.0030 i \\
-16.4908 \\
-15.8507 \\
-12.699 \\
-6.0837 \pm 5.5110 i \\
0.5212 \pm 2.7935 i \\
-3.7639 \pm 0.0046 i \\
-1.9311 \\
-0.2654 \\
-1.0596 \\
-0.8994\end{array}$ & Subject 6 & $\begin{array}{l}-15.1371 \\
-14.5957 \\
-11.8858 \pm 0.0833 i \\
-11.4523 \\
-5.4959 \pm 5.0598 i \\
0.5283 \pm 2.5826 i \\
-4.9195 \\
-3.3701 \\
-1.7174 \\
-0.2807 \\
-1.1862 \\
-0.9736\end{array}$ & $\begin{array}{l}-12.8123 \\
-12.2946 \\
-11.6181 \pm 0.0342 i \\
-9.8398 \\
-4.6251 \pm 4.4009 i \\
-5.9136 \\
0.5271 \pm 2.2633 i \\
-2.7977 \\
-1.4269 \pm 0.0797 i \\
-0.3149 \\
-0.824\end{array}$ \\
\hline Subject 3 & $\begin{array}{l}-15.0013 \pm 0.0114 i \\
-10.2558 \pm 1.7653 i \\
-10.537 \\
-4.5743 \pm 4.5595 i \\
0.5431 \pm 2.2911 i \\
-4.9766 \\
-3.1225 \\
-1.5874 \\
-1.2029 \\
-0.3013 \\
-0.6783\end{array}$ & $\begin{array}{l}-13.7419 \pm 0.0090 i \\
-8.7362 \pm 1.5372 i \\
-8.9667 \\
-3.8540 \pm 3.9678 i \\
-6.2969 \\
0.5273 \pm 2.0083 i \\
-2.6019 \\
-0.3464 \\
-1.4565 \\
-1.3821 \\
-1.0587\end{array}$ & Subject 7 & $\begin{array}{l}-12.4799 \pm 2.6892 i \\
-12.6840 \pm 0.0420 i \\
-11.4149 \\
-5.8487 \pm 5.6597 i \\
0.5437 \pm 2.7731 i \\
-4.4705 \\
-4.2271 \\
-2.2759 \\
-0.257 \\
-1.0143 \\
-0.7352\end{array}$ & $\begin{array}{l}-11.9457 \pm 0.0425 i \\
-10.3555 \pm 2.3502 i \\
-9.1755 \\
-4.8210 \pm 4.8669 i \\
0.5509 \pm 2.4010 i \\
-4.9047 \\
-3.7206 \\
-1.8938 \\
-1.1786 \\
-0.2814 \\
-0.5911\end{array}$ \\
\hline Subject 4 & $\begin{array}{l}-17.6777 \pm 0.0111 i \\
-11.5090 \pm 2.1429 i \\
-11.5066 \\
-5.2304 \pm 5.1113 i \\
0.5474 \pm 2.5428 i \\
-4.2194 \\
-3.6982 \\
-1.8838 \\
-0.2755 \\
-1.0146 \\
-0.7408\end{array}$ & $\begin{array}{l}-17.1681 \pm 0.0079 i \\
-9.9828 \pm 1.9590 i \\
-9.8461 \\
-4.5134 \pm 4.5514 i \\
0.5453 \pm 2.2759 i \\
-4.7514 \\
-3.1888 \\
-1.6225 \\
-1.1463 \\
-0.3003 \\
-0.634\end{array}$ & & & \\
\hline
\end{tabular}

Table 5.3: Eigenvalues for Subjects 1 to 7 in the standing and supine positions. 


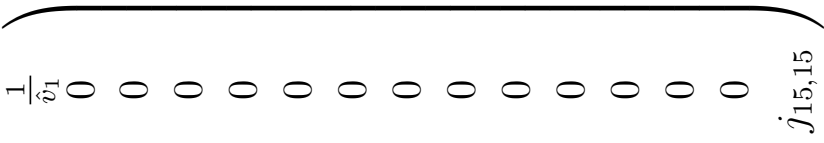

$$
\begin{aligned}
& 00000000000000 \frac{1}{-\left.\right|^{2}}
\end{aligned}
$$

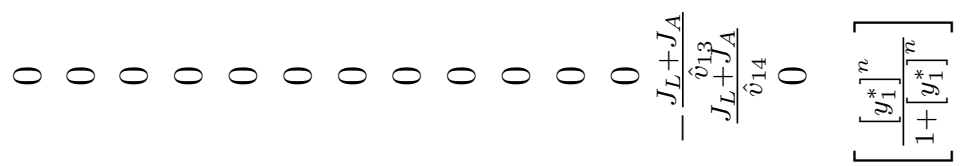

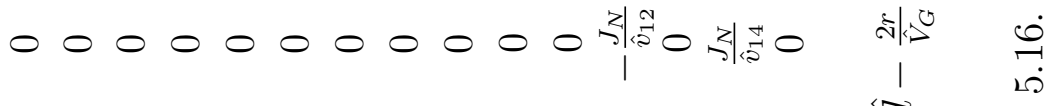

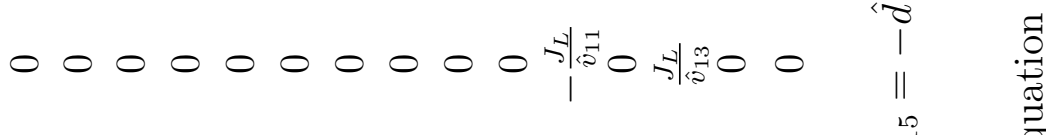

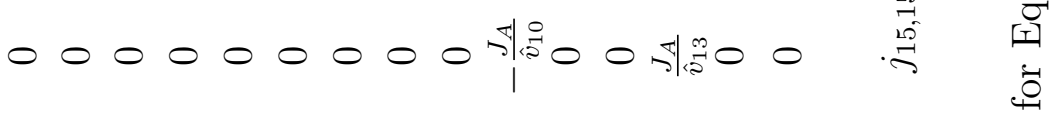

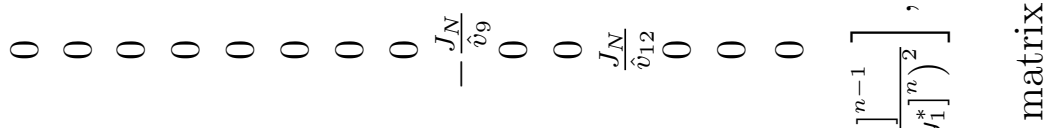

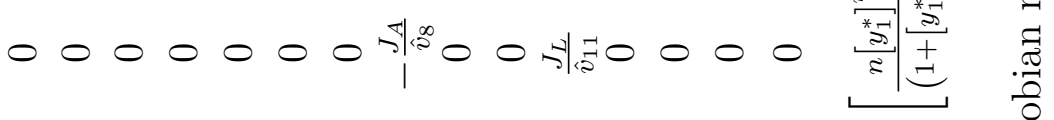

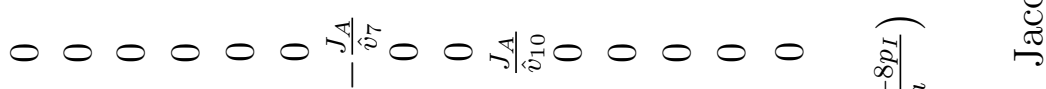

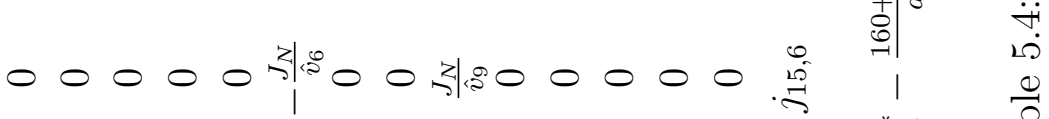

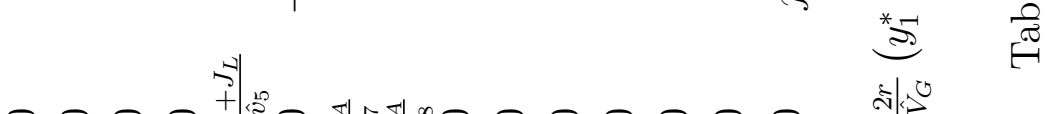

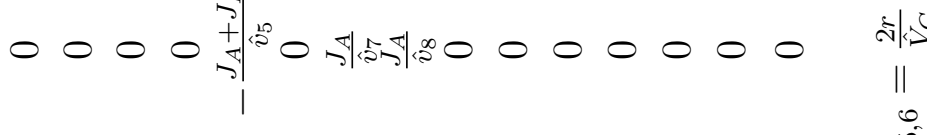

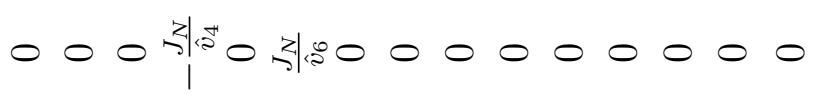

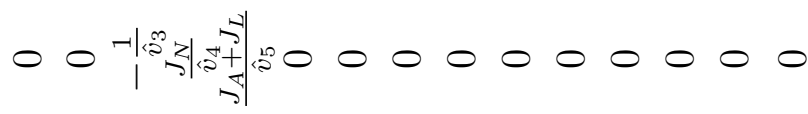

$$
\begin{aligned}
& 0-1 / 2 \pi-1,0000000000 \\
& -1 / 2-1,50000000000000
\end{aligned}
$$

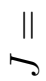




\subsection{Sensitivity Analysis}

Sensitivity analysis was performed on the model parameters found above. Eighteen parameters $\left(V_{G}, p_{I}, m, v_{T}\right.$, and $v_{i}$ for $\left.i=1, \ldots, 14\right)$ played a key role in determining the position of the Hopf bifurcation curve in the $(r, \mu)$ plane, and thus analysis was performed on these individually.

\subsubsection{Increasing and Decreasing Parameters By 30\%}

In order to determine the sensitivity of each parameter in the system, first a reasonable range of values for the ventilation-perfusion ratio $(r)$ was chosen (0.6 to 1.6) [25]. The Hopf bifurcation curve was plotted on the $(r, \mu)$ plane for this range of $r$. Typical parameter values listed in Appendix B were chosen to be the base case parameters for analysis. Next, a percentage $(30 \%)$ of the parameter was added and subtracted from the base case, where three curves were plotted, one for each value of the parameter, $-30 \%$, base, $+30 \%$. In order to determine how sensitive each parameter is, the area between the positive curve and base case was calculated over the whole range of $r, 0.6<r<1.6$, along with the area between the negative curve and base case. Resulting graphs can be seen in Figures 5.3, 5.4, and 5.5.

Results show that overall the model is not very sensitive. $V_{G}, V_{T}, v_{1}, v_{2}, v_{3}, v_{4}$, and $v_{6}$ are the most sensitive parameters in the system. Increasing $V_{G}$ and $v_{3}$ shift the curve up relative to the base case where as decreasing $V_{T}, v_{1}, v_{2}, v_{4}$, and $v_{6}$ will cause the curve to shift up. All other parameters $\left(v_{5}, v_{7-14}, m, p_{I}\right)$ are not sensitive and thus cause little to no shift in the curve. Table 5.5 lists the areas between the bifurcation curves in the $(r, \mu)$ plane for the different parameters. From these results it is expected that there will be small changes between curves. 

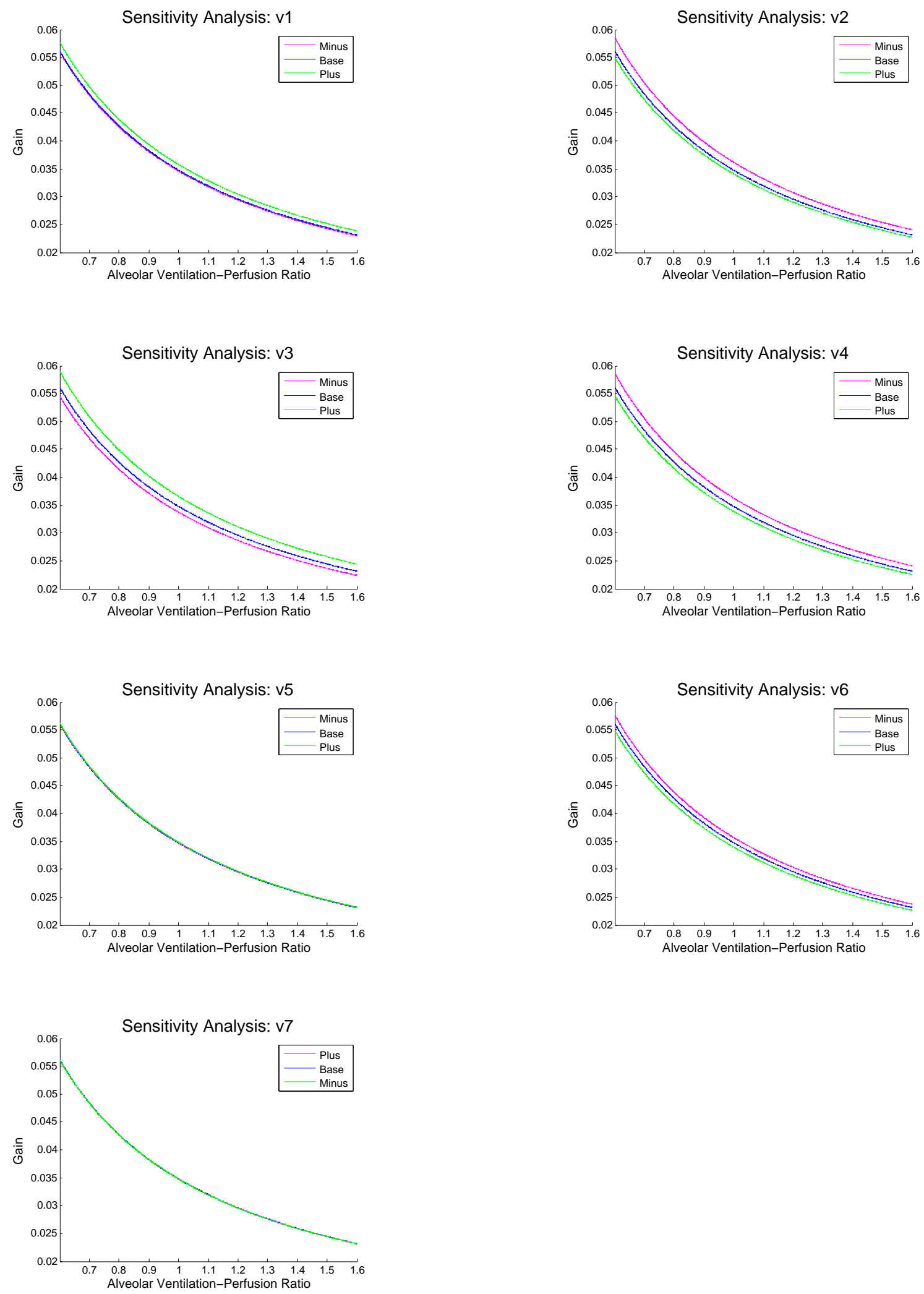

Figure 5.3: Sensitivity analysis fluid shift model. Part 1: parameters $v_{1}$ to $v_{7}$. 

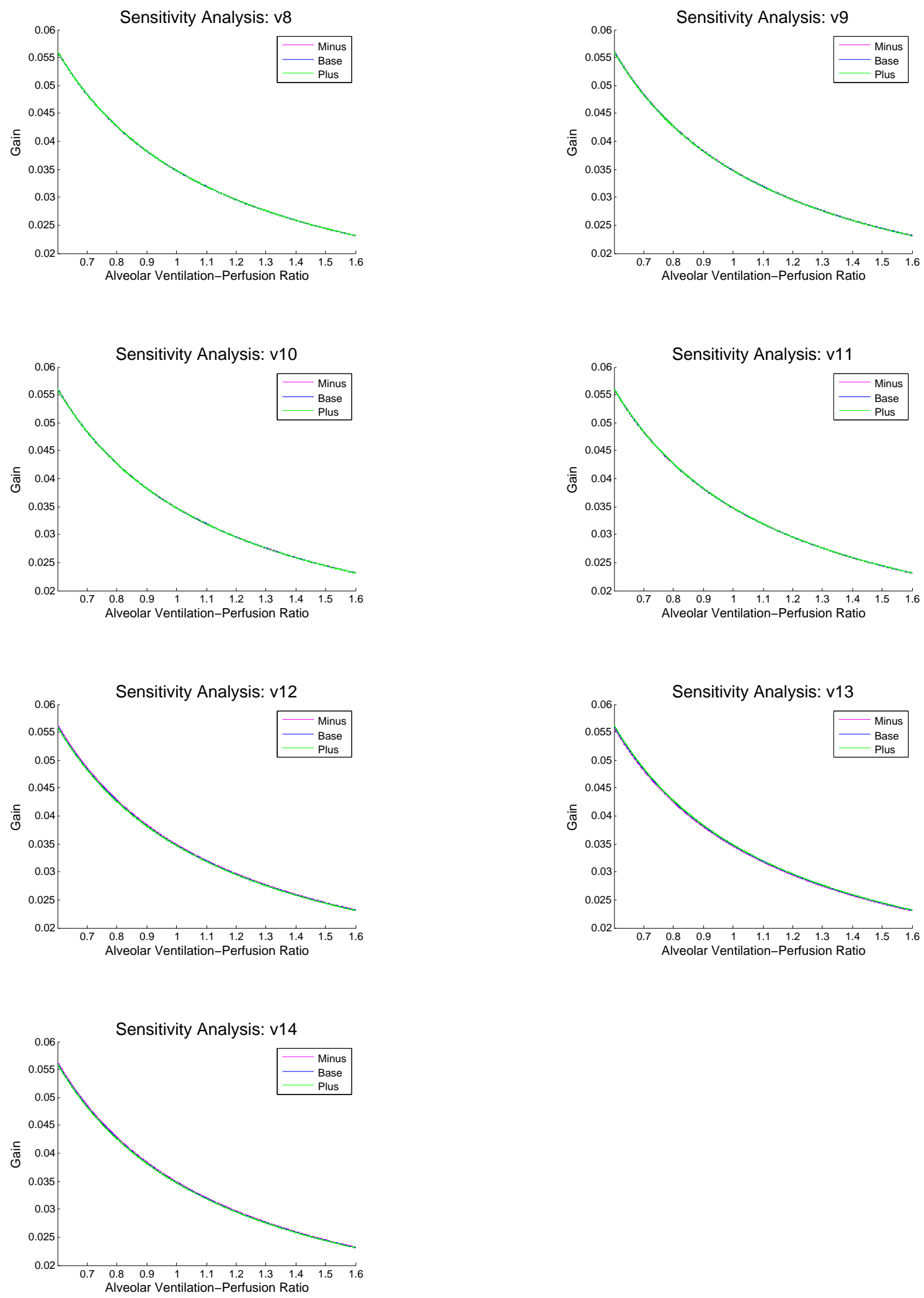

Figure 5.4: Sensitivity analysis fluid shift model. Part 2: parameters $v_{8}$ to $v_{14}$. 

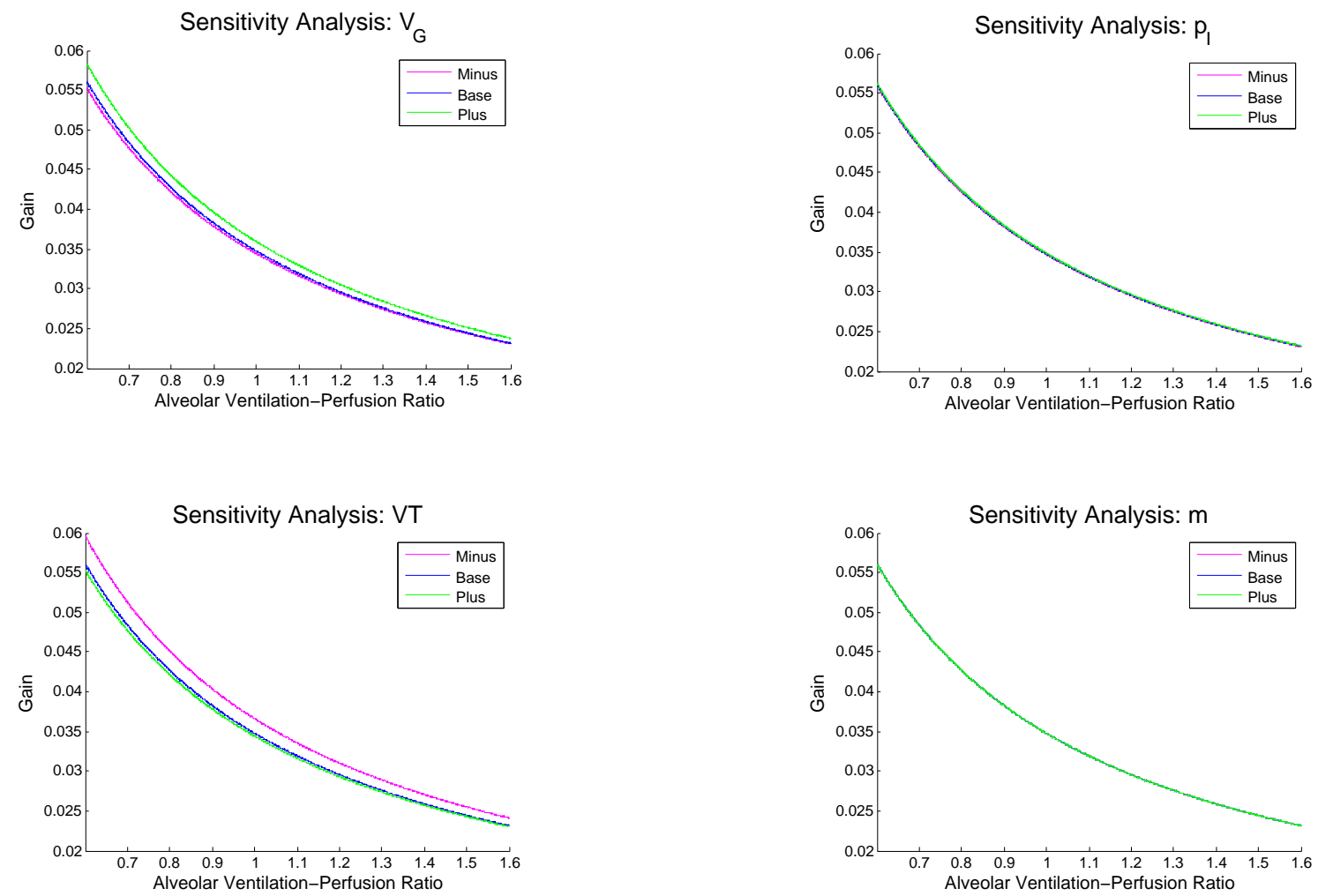

Figure 5.5: Sensitivity analysis fluid shift model. Part 3: parameters $V_{9}, p_{I}, V_{T}$, and $m$. 


\begin{tabular}{|c|ccc|}
\hline Parameter & Area Minus & Area Plus & Total Area \\
\hline$V_{G}$ & $-3.2251 \mathrm{e}-04$ & 0.0012 & 0.0015 \\
$p_{I}$ & $-7.6533 \mathrm{e}-05$ & $7.6616 \mathrm{e}-05$ & $1.5315 \mathrm{e}-04$ \\
$v_{T}$ & 0.0018 & $-3.5572 \mathrm{e}-04$ & 0.0022 \\
$v_{1}$ & $-1.5779 \mathrm{e}-04$ & $9.9926 \mathrm{e}-04$ & 0.0012 \\
$v_{2}$ & 0.0014 & $-6.6588 \mathrm{e}-04$ & 0.0021 \\
$v_{3}$ & -0.0010 & 0.0018 & 0.0028 \\
$v_{4}$ & 0.0015 & $-8.8836 \mathrm{e}-04$ & 0.0024 \\
$v_{5}$ & $4.7538 \mathrm{e}-05$ & $5.5266 \mathrm{e}-05$ & $1.0280 \mathrm{e}-04$ \\
$v_{6}$ & $9.1409 \mathrm{e}-04$ & $-7.7713 \mathrm{e}-04$ & 0.0017 \\
$v_{7}$ & $9.2516 \mathrm{e}-06$ & $-8.6871 \mathrm{e}-06$ & $1.7939 \mathrm{e}-05$ \\
$v_{8}$ & $-4.4471 \mathrm{e}-06$ & $4.7777 \mathrm{e}-06$ & $9.2248 \mathrm{e}-06$ \\
$v_{9}$ & $2.7110 \mathrm{e}-05$ & $-2.6812 \mathrm{e}-05$ & $5.3922 \mathrm{e}-05$ \\
$v_{10}$ & $9.2516 \mathrm{e}-06$ & $-8.6871 \mathrm{e}-06$ & $1.7939 \mathrm{e}-05$ \\
$v_{11}$ & $-1.7876 \mathrm{e}-05$ & $1.1619 \mathrm{e}-05$ & $2.9495 \mathrm{e}-05$ \\
$v_{12}$ & $1.3689 \mathrm{e}-04$ & $-5.6483 \mathrm{e}-05$ & $1.9338 \mathrm{e}-04$ \\
$v_{13}$ & $-1.3163 \mathrm{e}-04$ & $8.1619 \mathrm{e}-05$ & $2.1325 \mathrm{e}-04$ \\
$v_{14}$ & $1.3966 \mathrm{e}-04$ & $-5.6153 \mathrm{e}-05$ & $1.9582 \mathrm{e}-04$ \\
$m$ & 0 & 0 & 0 \\
\hline \multicolumn{4}{|r}{}
\end{tabular}

Table 5.5: Area between base curve and curve produced by shifting each parameter by $\pm 30 \%$ using standing parameter values from Subject 1 (male).

\subsection{Results}

\subsubsection{Experimental Data}

Experiments for two different body positions (upright, recumbent) were examined in seven typical adult subjects (two males and five females) who do not have CSR or any other oscillatory breathing pattern. Prior to measurements, subjects had been in the upright position for more than five hours. Electrodes were placed in multiple locations along the right side of the body were bioelectrical impedance was used to measure resistances, which were later converted to fluid volumes [37]. Measurements were first taken while each subject stood for five minutes; fluid volumes were recorded at both time zero and after five minutes. Next, subjects entered the supine position for a 90 minute duration. Fluid volumes were once again recorded at time zero and after 90 minutes. The purpose was to determine where fluid tends to shift upon entering the 
recumbent position, with hopes of understanding if this shift in fluid plays a key role in the onset of CSR. Measured fluid volumes were of extracellular fluid (i.e., blood and intracellular fluid) so in order to minimize the effects of intracellular fluid volumes and to account for error in measurements, total amounts of fluid in each subject in respective body positions was normalized to five litres. Normalized blood volumes for each subject can be found in Table 6.4.

\subsubsection{Body Position}

Figures 5.7 and 5.9 show resulting Hopf bifurcation curves for standing at time zero $\left(\right.$ standing $\left._{0}\right)$ and after five minutes $\left(\right.$ standing $\left._{5}\right)$ as well as curves for supine at time

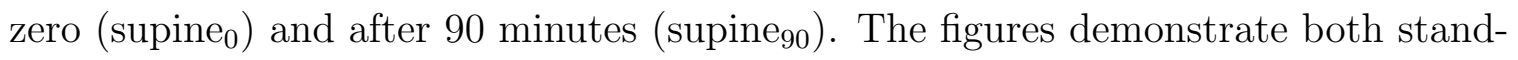
ing curves are essentially in the same position (curves are indistinguishable). Thus although some fluid does shift into the legs after standing for five minutes, it is clear that it is not a significant amount and curves are essentially identical, as expected. Supine curves ( supine $_{0}$ and supine gn ) have slightly different positions depending on the subject. For some subjects (ie. Subject 1) curves in the supine position are essentially equal. Immediately after changing body position, there is an obvious downward shift in the curve. After 90 minutes, the curve experiences a relatively small shift downward. Conversely, Subject 2 has little fluid shift upon immediately entering the recumbent position, however, after 90 minutes a relatively large downward shift has occurred.

Key results come when comparing standing 5 and supine $_{90}$, where it is assumed that the standing volume measured after five minutes is an accurate representation of fluid placement while upright. Fluid shift is known to have an exponential decay up until 120 minutes [12], so in a similar way, assume that after remaining in the supine position for 90 minutes, fluids have reached an equilibrium and remain stable for the remainder. Therefore we can best compare body position by looking specifically at 
the curves produced from standing 5 and supine 90 . Results are shown in Figures 5.8 and 5.10 .

Gender differences play a large role in the position of the curves and will be described below, however for body position purposes we look at all seven subjects. By examining Figures 5.8 and 5.10, it is clear that the Hopf curve produced at supine ${ }_{90}$ is positioned lower than the curve produced at standing 5 . This means that in the supine position, the steady region (stable breathing pattern) has decreased. This is true for all seven subjects; the supine curve falls below the standing curve, with differences in curves and their respective position being attributed to the effect of gender (discussed in section 5.7.2). In conclusion the model shows that an individual, regardless of gender, is more likely to experience Cheyne-Stokes Respiration in the supine position than the standing position, which supports our hypothesis.

\section{Hill Function}

The Hill function was used as the control for the system. To determine how the control was affected when body position changed, the ventilation-perfusion ratio $(r)$ was fixed and the gain $(\mu)$ values were computed based on where that fixed value intersected the Hopf bifurcation curve. Here, $r$ was chosen to equal one. Corresponding $\mu$ values obtained from intersecting both the standing ${ }_{5}$ curve and the supine ${ }_{90}$ curve are shown in Table 5.6. From the Hill function itself, it is known that increasing the gain results in an increase in the sensitivity of the control function. Results show that the gain is higher in the standing position than in the supine position. Since an increase in the gain corresponds to an increase in the sensitivity of the chemoreceptors, results show that chemoreceptors are more sensitive to changes in $\mathrm{CO}_{2}$ in the upright position, suggesting a decreased likelihood of CSR.

Changes in the location of the Hopf curve due to fluid shift can be described as equivalent changes in the gain, for a fixed ventilation-perfusion ratio. Differences in 


\begin{tabular}{cccc}
\hline Subject & Standing $_{5}$ Gain & Supine $_{90}$ Gain & Absolute Difference \\
\hline Subject 1 & 0.0349 & 0.0327 & 0.0022 \\
Subject 2 & 0.0369 & 0.0348 & 0.0021 \\
Subject 3 & 0.0321 & 0.0315 & 0.0006 \\
Subject 4 & 0.0328 & 0.0319 & 0.0009 \\
Subject 5 & 0.0335 & 0.0328 & 0.0007 \\
Subject 6 & 0.0340 & 0.0330 & 0.0010 \\
Subject 7 & 0.0336 & 0.0321 & 0.0015 \\
\hline
\end{tabular}

Table 5.6: Gain values on the Hopf bifurcation curve resulting from a fixed $r$ value $(r=1)$ in the standing position after five minutes and supine position after 90 minutes.

gain values between the standing and supine positions resulting from fluid shift (Table 5.6) for each subject appear to be small. Substituting these values into the control function display differences between curves (Figure 5.6). Resulting curves in both the standing and supine positions are so similar, they are practically indistinguishable. Thus it can be concluded that the change in body position does not have a large effect on the value of the gain. Biologically this can be interpreted as the change in fluid volume has a limited effect on determining the likelihood of causing CSR compared to a change in the control function. Simply changing the gain of the system (due to a stroke, congestive heart failure, atrial fibrillation) will result in a much larger or smaller likelihood of developing CSR compared to body position. CheyneStokes Respiration is primarily neurological in origin and most prevalent during sleep, therefore these results fit well with biological data.

\subsubsection{Gender}

\section{Male Analysis}

Hopf bifurcation curves that result from entering the normalized fluid volume data for male subjects 1 and 2 into our model can be seen in Figures 5.7 and 5.8. The purple curve in Figure 5.8 represent the Hopf curve produced from fluid volumes in the standing position after five minutes while the blue curve represents the supine 
(a)

(c)

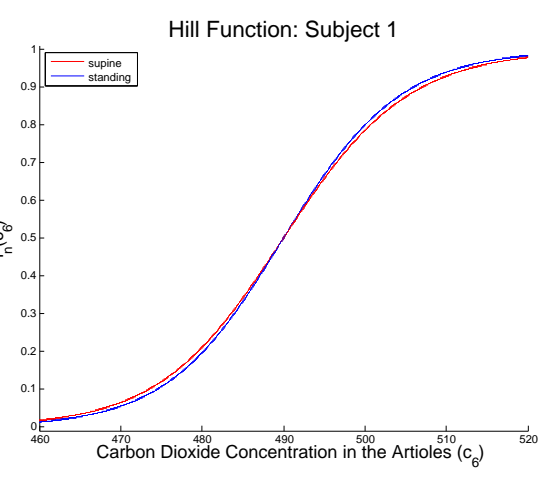

(e)

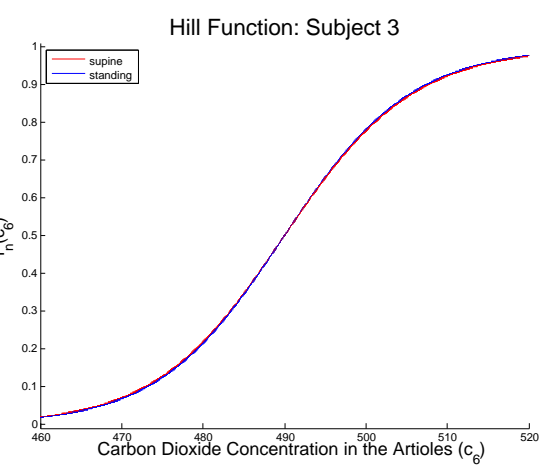

(g)
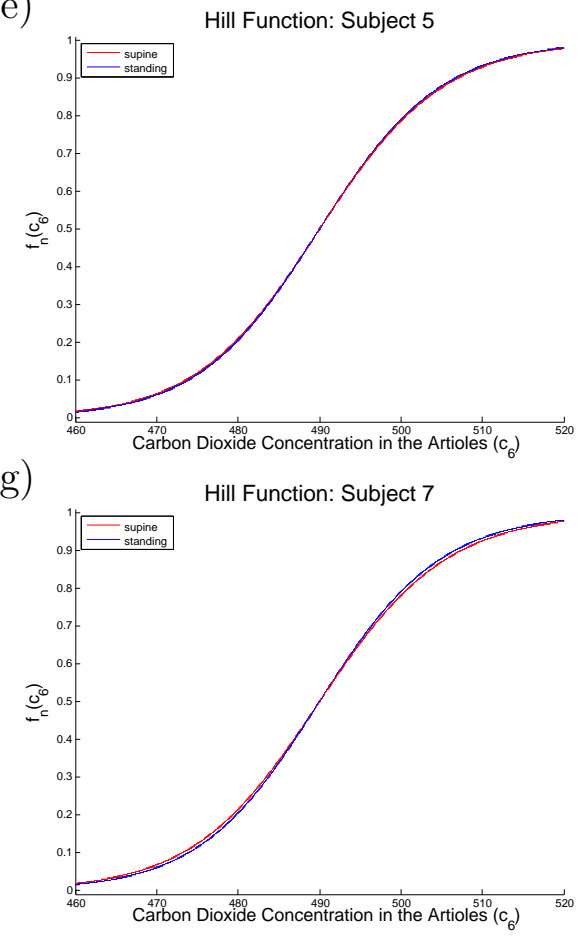

(b)

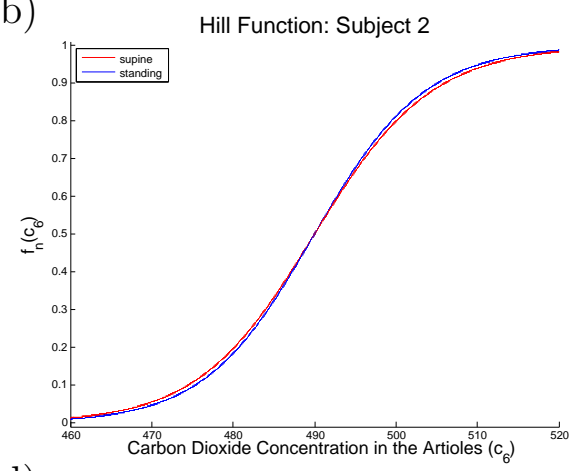

(d)

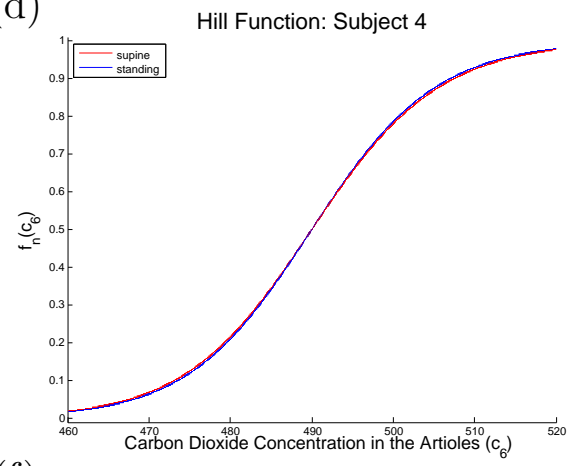

(f)

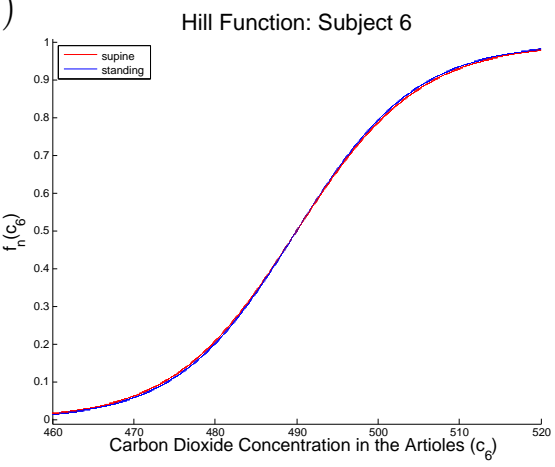

Figure 5.6: Equivalent changes in the Hill function due to Hopf curve location for both the standing 5 and supine 90 curves. (a), (b) male subjects, (c), (d), (e), (f), (g) female subjects. 
(a)

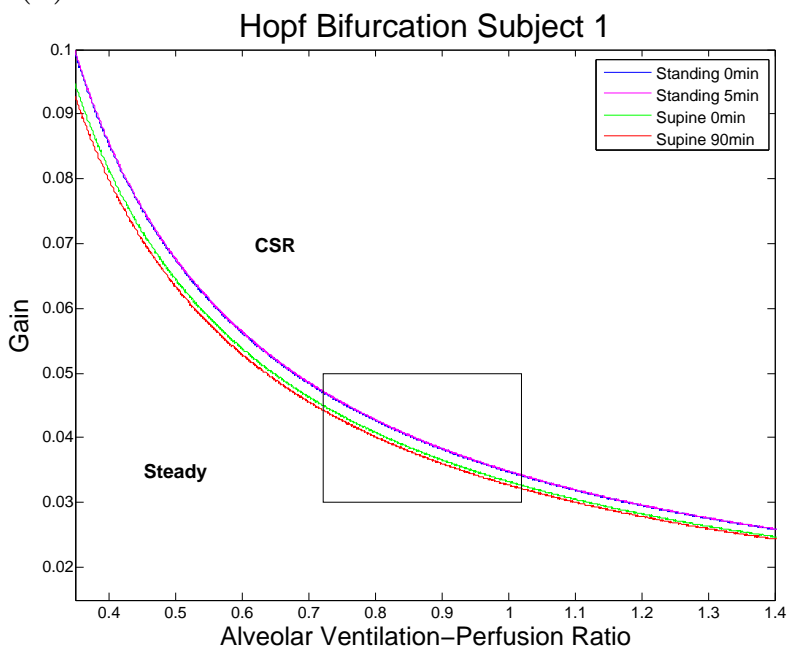

(b)

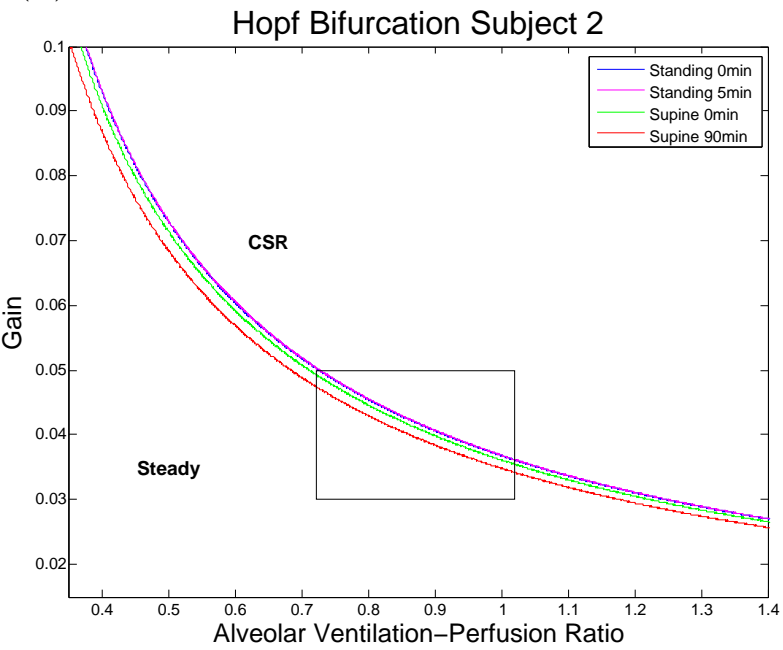

Figure 5.7: Hopf bifurcation curves for male subjects 1 and 2. Standing 0,5 and supine $_{0,90}$. Boxes represent a range of typical $r$ values.

(a)

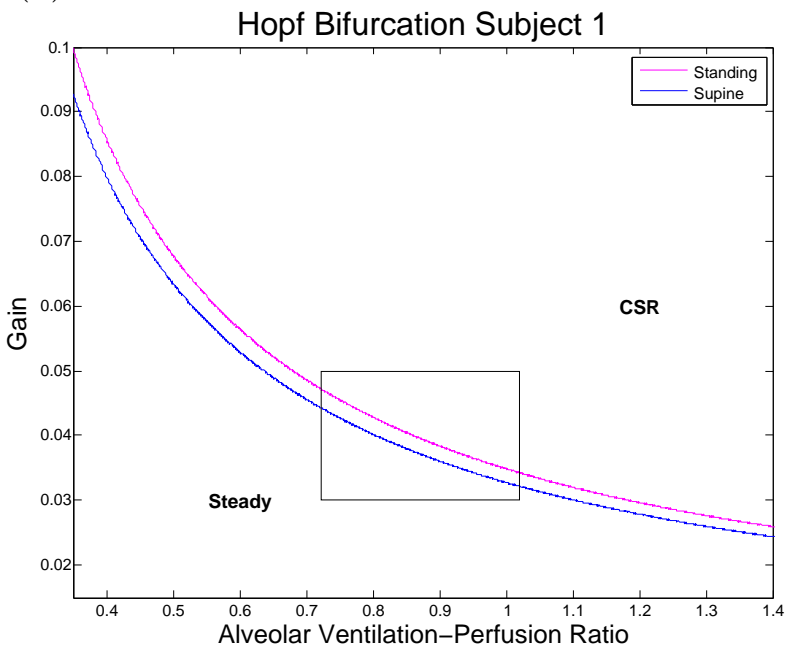

(b)

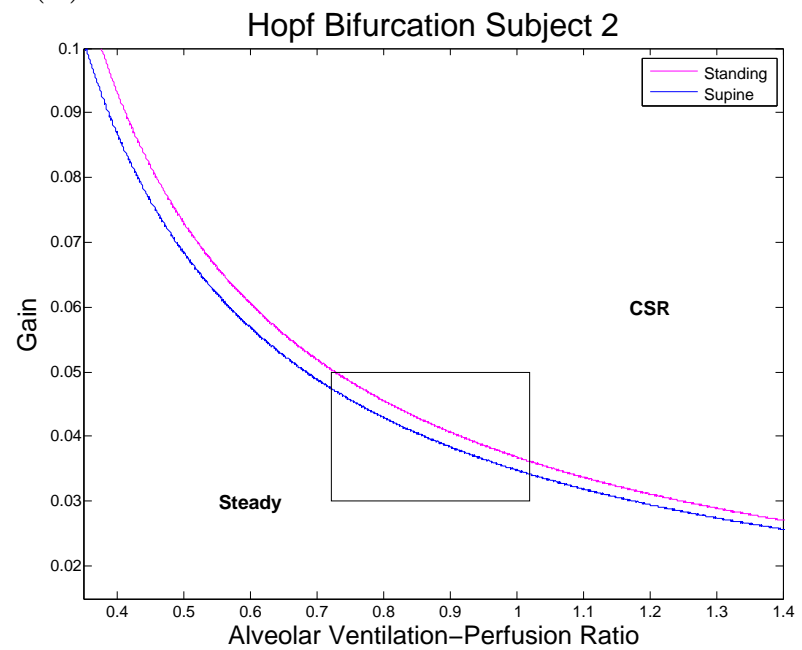

Figure 5.8: Hopf bifurcation curves for male subjects 1 and 2. Standing St supine $_{90}$. Boxes represent a range of typical $r$ values. 
position after 90 minutes. As stated above, we can assume that using standing ${ }_{5}$ and supine $_{90}$ provides the best representation of fluid shift from the upright to the recumbent position at anytime during sleep.

Typical gain and ventilation-perfusion ratio values for adult male parameters lie in the area below both the standing and supine curves, in the region labeled 'steady'. This area represents where a typical individual will experience steady breathing patterns. As parameter values change to the point where they cross the curve, breathing patterns change from stable to unstable, representing oscillatory breathing (CSR). Figures 5.7 and 5.8 clearly show that the supine curve falls below the standing curve. This indicates that in the supine position the steady region is smaller than it is in the standing position, suggesting individuals are more likely to experience CSR while supine. This result gives strong evidence for the hypothesis that as fluid shifts from the legs to the neck, individuals are more likely to experience CSR.

Assuming a ventilation-perfusion ratio of one, the gain values that lie on the Hopf curve for both subjects are shown in Table 5.6. From the standing to supine position, gain values decrease by approximately 0.002 . Although this does not provide a significant change in the control function, seen in Figure 5.6 (a) and (b), it is a good measure of sensitivity. Using parameter values from Subject 1, the gain value on the curve in the standing position was 0.0349 , while in the supine position it was 0.0327 , with an absolute difference of 0.0022 . Subject 2 parameter values resulted in gain values of 0.0369 and 0.0348 on the Hopf curve in the standing and supine positions respectively, generating an absolute difference of 0.0021. Data from both subjects resulted in similar gain values and differences, with supine curve values being less than standing values. This suggests that male subjects are more sensitive to changes in $\mathrm{CO}_{2}$ concentration in the upright position. 


\begin{tabular}{ccccc}
\hline Subject & Leg (mL) & Abdomen $(\mathbf{m L})$ & Chest $(\mathbf{m L})$ & Neck $(\mathbf{m L})$ \\
\hline 1 & -45 & 61 & -94 & 78 \\
2 & -245 & -15 & 221 & 39 \\
\hline
\end{tabular}

Table 5.7: Male body fluid shift: Supine - Standing Volumes.

\begin{tabular}{ccccc}
\hline Subject & Leg $(\mathbf{m L})$ & Abdomen $(\mathbf{m L})$ & Chest $(\mathbf{m L})$ & Neck $(\mathbf{m L})$ \\
\hline 3 & -470 & 68 & 346 & 56 \\
4 & -296 & 19 & 228 & 50 \\
5 & -311 & 48 & 219 & 44 \\
6 & -382 & 21 & 320 & 41 \\
7 & -365 & 54 & 238 & 73 \\
\hline
\end{tabular}

Table 5.8: Female body fluid shift: Supine - Standing Volumes.

\section{Female Analysis}

Hopf bifurcation curves for female subjects $(3,4,5,6$, and 7$)$ were produced in the same way as stated above and are shown in Figure 5.9. Although the curves lie almost on top of one another, upon close examination, supine curves fall below the standing curves. This means that females are also more likely to experience CSR in the supine position because steady regions become smaller in the recumbent position. The black box represents typical ventilation-perfusion values (0.3 to 2.1) [43] for a range of typical gain values (0.01 to 0.1 ).

In order to get a better understanding of the differences between curves, the gain of each curve at $r=1$ was calculated. These were input into the control function and the output was analyzed. Considering only standing ${ }_{5}$ and supine $_{90}$, the gain values are listed in Table 5.6. Absolute difference are approximately 0.0009 which is reflected in the control function seen in Figure 5.6 (c), (d), (e), (f), and (g). Control curves are virtually indistinguishable; body position does not change the control function. Changes in gain values are a significantly stronger determining factor than body position. It can then be concluded that females are more likely to experience CSR in the recumbent position however the difference in the likelihoods between body 
positions is diminutive.

\section{Male versus Female}

The predominant difference between males and female lies in the distance between curves, experimental fluid shift, and the relative change in gain values. For explanation purposes, consider only standing 5 and supine $_{90}$ as they are the best representation of fluid shift. In female subjects, curves lie very close to one another as opposed to curves for male subjects which display a distinct difference. As stated in the analysis above, both males and females are more likely to experience unstable breathing patterns in the supine position. Although this hold true for females, overall they are less likely to experience CSR than males.

The differences in experimental fluid shift data are shown in Table 5.7 and 5.8 for male and female subjects respectively. Male and female data display different behaviour in fluid shifts which can be seen in the position of respective Hopf curves. Female data appears to be consistent between subjects while male data appears to be highly inconsistent, particularly in the abdomen and chest body regions where the addition or loss of fluid is dependent on the subject. From these measurements, and from sensitivity analysis results in which the most sensitive parameters are located in the neck region, it appears as though the chest and abdomen volumes do not affect the Hopf curve substantially. In male subjects, initially the neck volume appears to be the determining factor. Upon comparing male and female fluid shifts in the neck, it is clear that male subjects experience both the smallest and largest shifts in neck fluid volume. Thus, the neck alone cannot be the only determining factor in the position of the Hopf curve. Parameter values corresponding to the chest region also display some sensitivity, however summing up the chest and neck fluid shifts in Tables 5.7 and 5.8 does not fully explain the differences between males and females either. Consequently, the neck, chest and abdomen volumes combined appear to be 
(a)

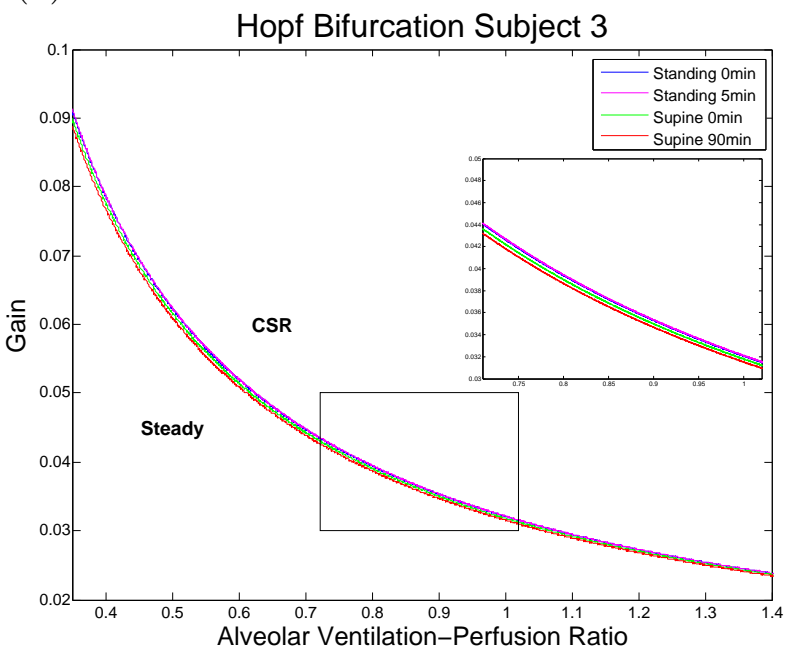

(c)

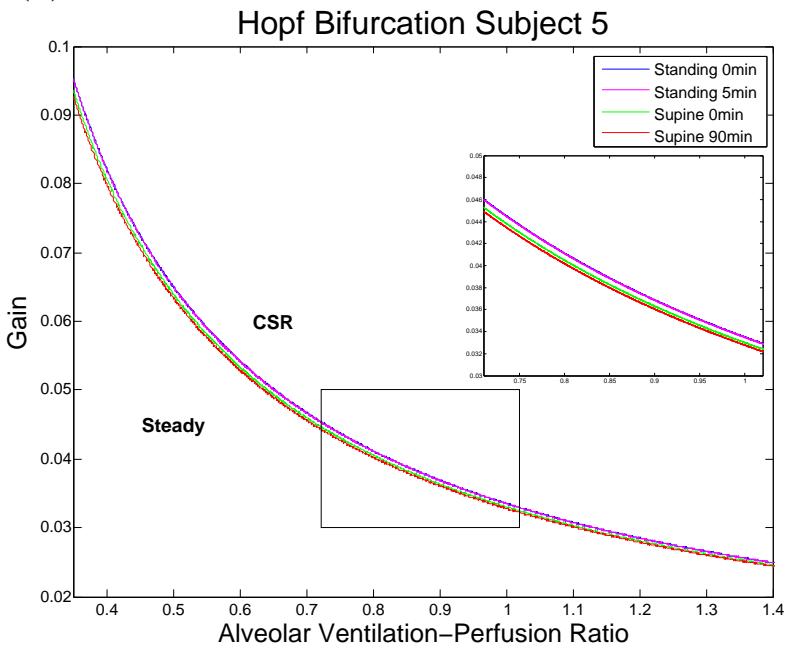

(e)

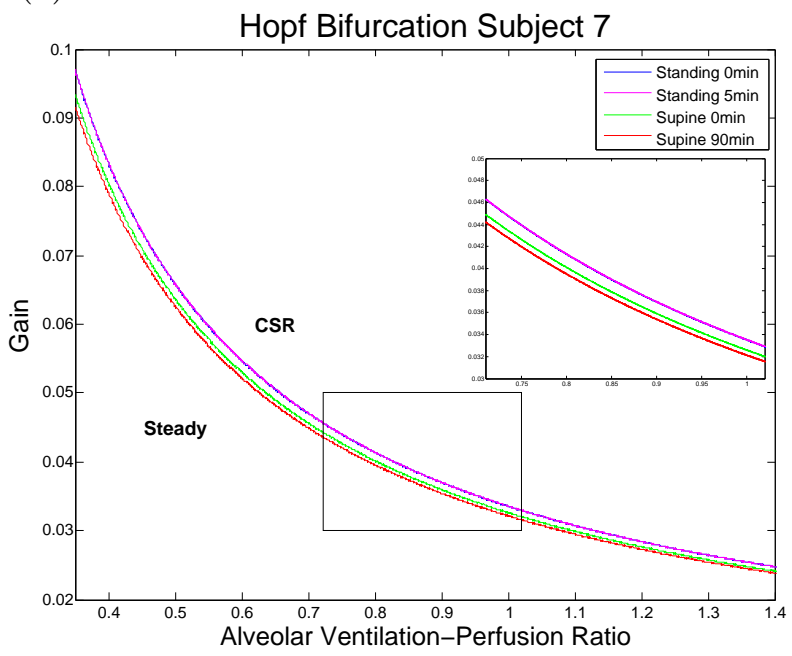

(b)

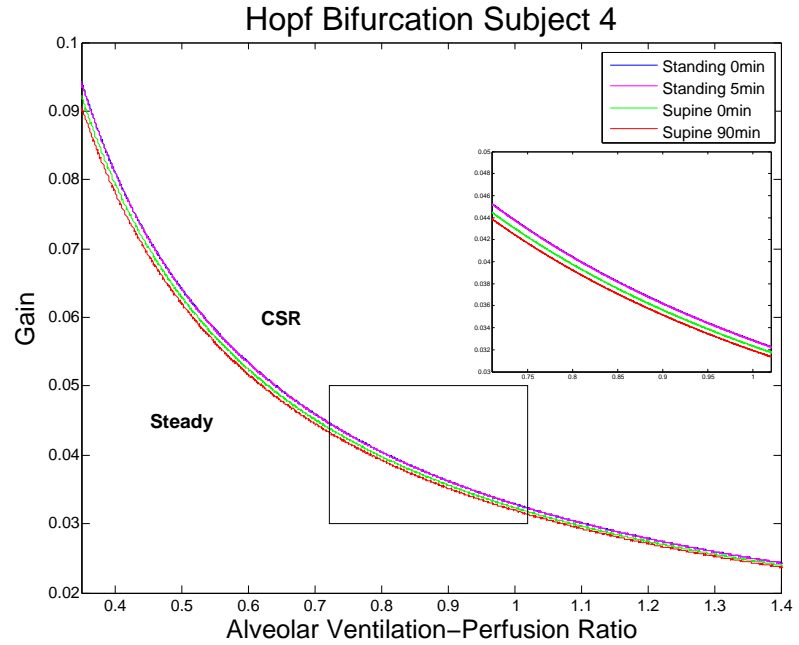

(d)

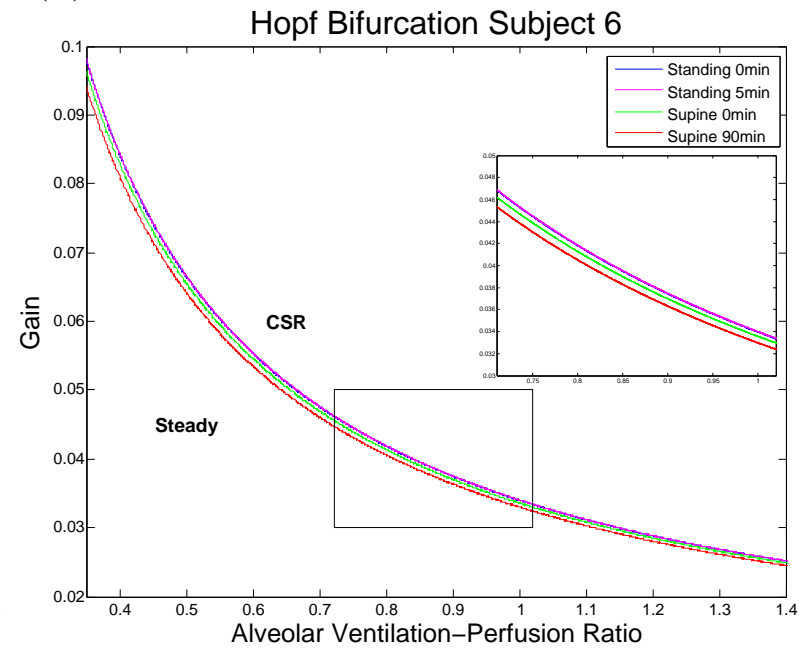

Figure 5.9: Hopf bifurcation curves for female subjects. Figures display all four body positions. Boxes represent typical gain and ventilation-perfusion values. 
(a)

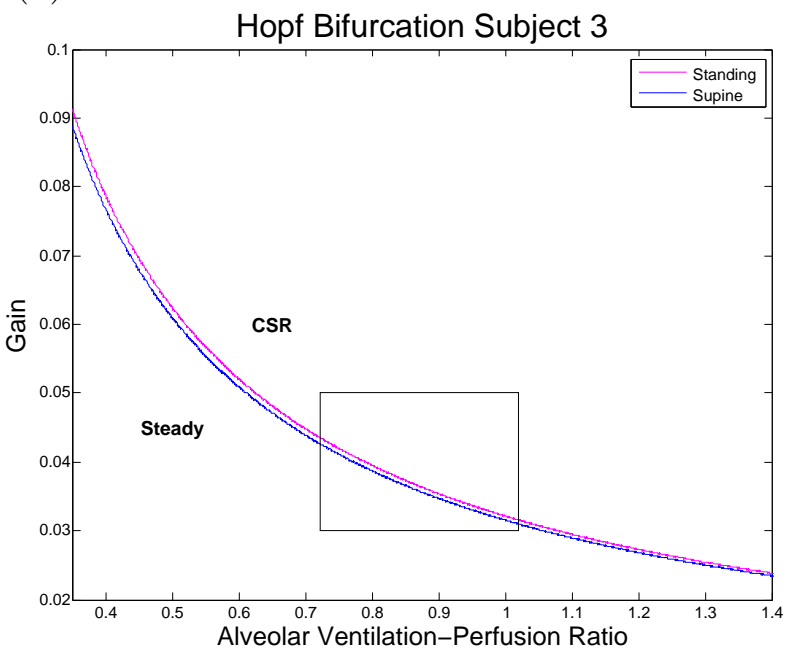

(c)

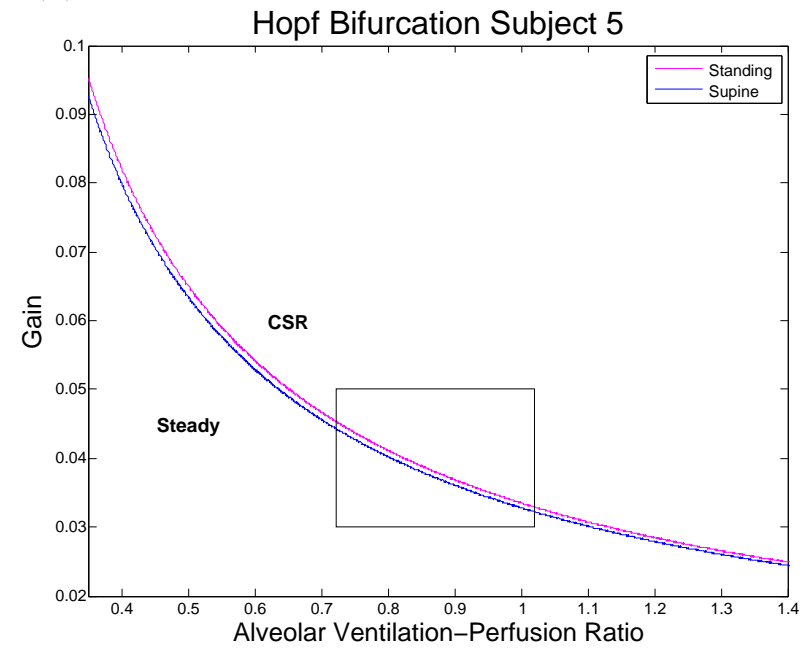

(e)

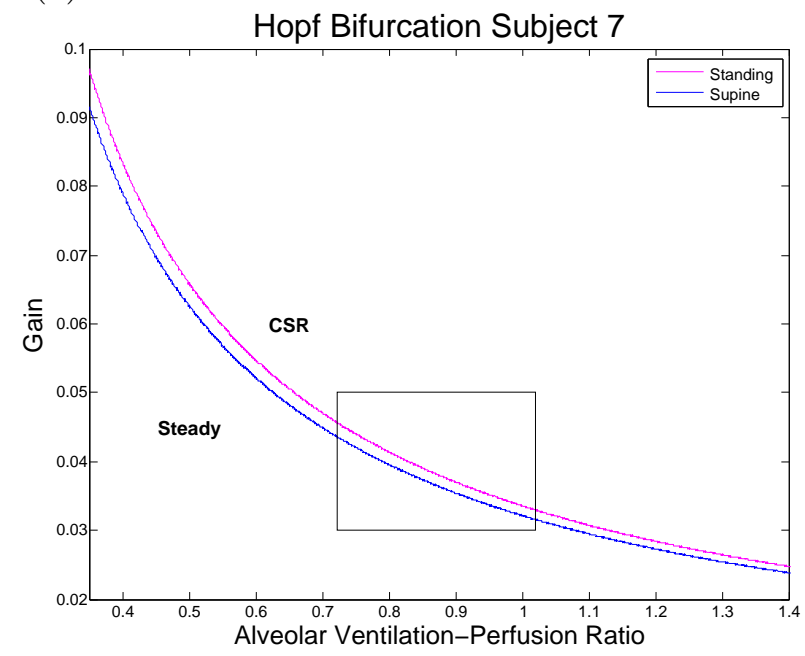

(b)

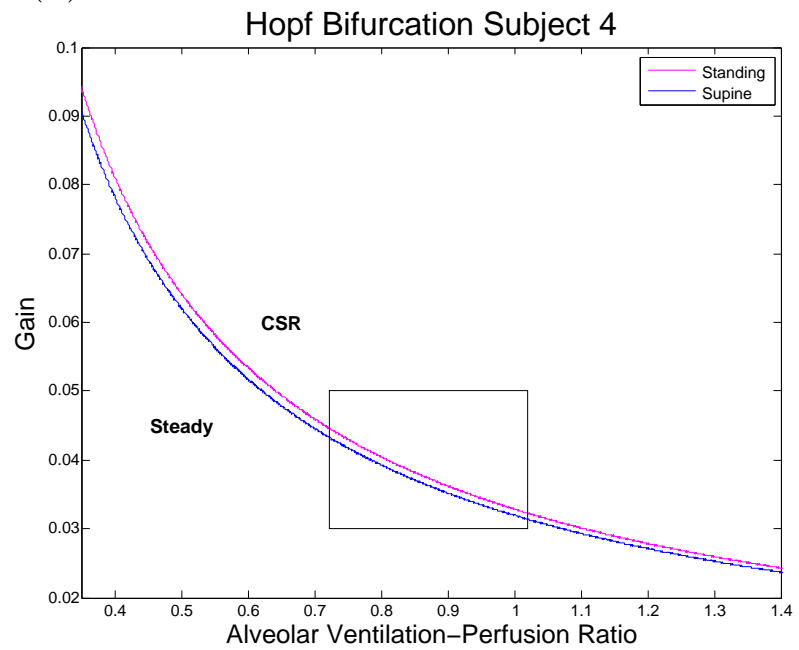

(d)

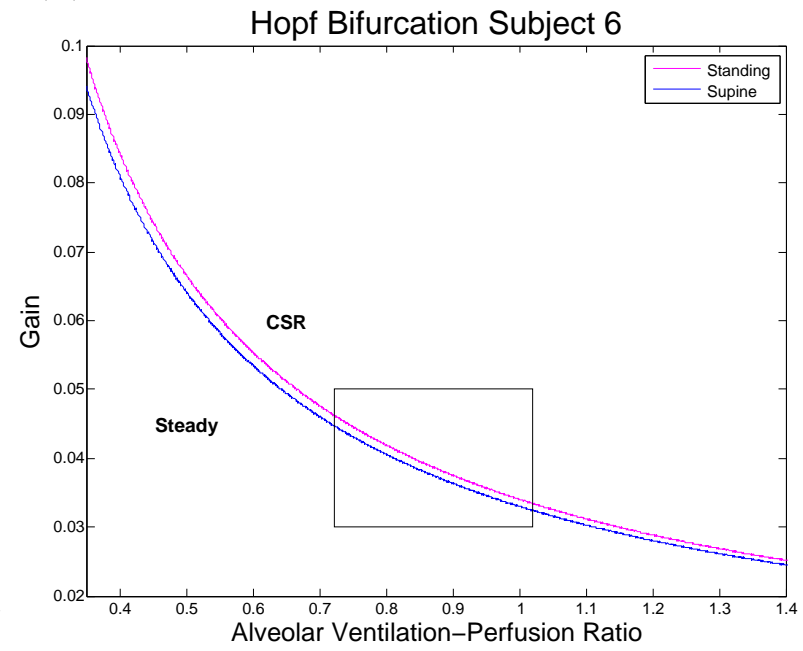

Figure 5.10: Hopf bifurcation curves for female subjects. Figures display standing at five minutes and supine after 90 minutes. Boxes represent typical ventilation-perfusion values. 
the determining factor in both the position of the Hopf curve and the fundamental differences between male and female likelihoods of experiencing CSR.

For ease of comparison, let us compare male and female Subjects 1 and 3. Assuming Subject 1 is a typical male in the standing position and holding $r$ constant, the gain value on the Hopf curve corresponding with $r=1$ is 0.0349 . In the supine position, the corresponding gain value from the supine curve is 0.0327 . There is a noticeable change in the position of the Hopf curve which is reflected in the differences in gain values. This change in the gain (although it is small) leads to a change in the control function. Therefore, typical parameter values fall very close to the supine 90 curve, so small changes in these values could lead to typical values falling on the unstable side of the curve, representative of CSR. Examining curves produced from Subject 3 parameter values in both the standing to supine positions, the gain values corresponding to $r=1$ experience little change (0.0321 standing, 0.0315 supine). Changes in both the Hopf curve and control functions are so minimal, females are only slightly more likely to experience CSR in the standing position as opposed to the supine position. Typical parameter values are no closer to the supine $e_{00}$ curve than they are to the standing ${ }_{5}$ curve, supporting the fact that females are less likely to experience CSR.

Overall observations give direct support for both hypotheses; males and females are more likely to experience CSR in the supine position than in the standing position, and males are more likely to develop CSR than females.

\subsection{Average Male and Female}

Individually, subjects display vastly different fluid shifts as well as body compositions (age, height, BMI). In an attempt to generalize the two male subjects and five female subjects into a population, the shape and results obtained from averaging the information is analyzed. 
When looking at male raw data, it appears as though each subject will display significantly different results. Table 5.7 shows the amount of fluid shifted measured when comparing supine $9_{0}$ volumes to standing ${ }_{5}$ volumes. The most surprising results occur in the abdomen and chest. Subject 1 gains fluid in the abdomen and loses it in the chest, while Subject 2 displays the exact opposite behaviour. Based on this data, it is expected that resulting curves from Subjects 1 and 2 will be significantly different. However, after averaging results (Figure 5.11 (a) and (b)), and comparing with individual curves it is clear that the curves are very comparable.

Gain values as listed above in the same relative region (standing or supine) are slightly different for each male subject, however, accounting for body size and other characteristics, curves are comparable. The resulting Hopf bifurcation curves from averaging blood volumes from Subjects 1 and 2 can be seen in Figure 5.11 where (a) displays all four results and (b) shows only standing ${ }_{5}$ and supine ${ }_{90}$. Therefore, despite differences in individual subjects, the resulting curves are similar to one another.

Female raw data does not have the same discrepancies as the males. In all 5 subjects the legs lose fluid when entering the recumbent position and the three other regions (abdomen, chest, neck) gain fluid (see Table 5.8). Further, the amount of fluid shifted in each region is comparable between subjects. Results for averaging female data can be seen in Figure 5.11 (c) and (d). As expected, the averaged curves are similar to individual results. Standing 0 and standing ${ }_{5}$ curve fall on top of one another with both supine $_{0}$ and supine $_{90}$ falling just below. The shift between curves seen in the average is relative to individual curves and shifts in relation to specific body characteristics (height, age, BMI). In conclusion, shifts in the curves produced from the average of female subjects are as expected and still give support of our hypothesis. 
(a)

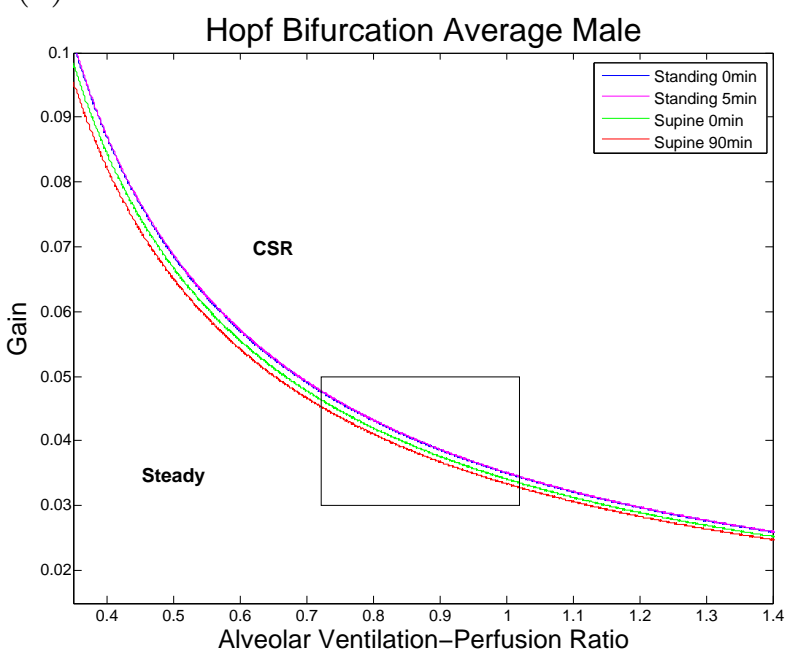

(c)

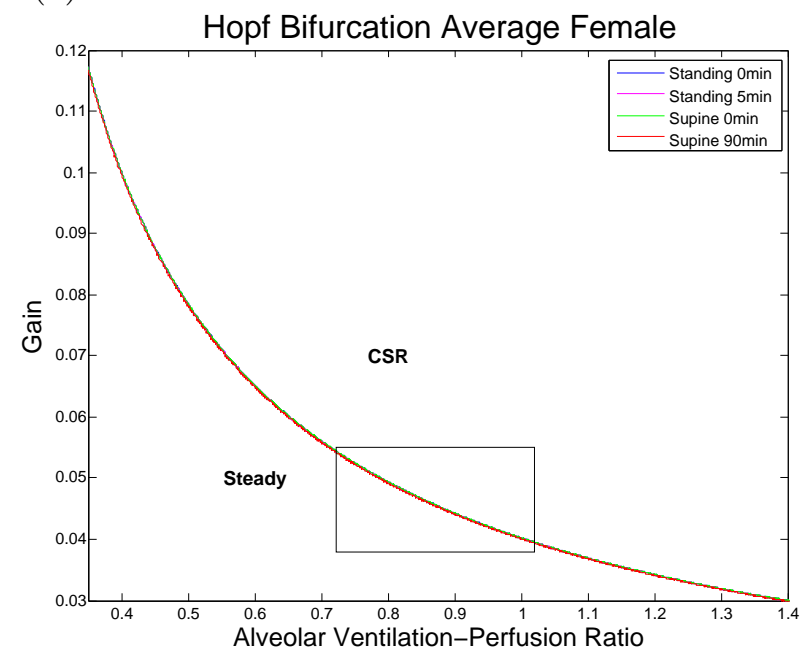

(b)

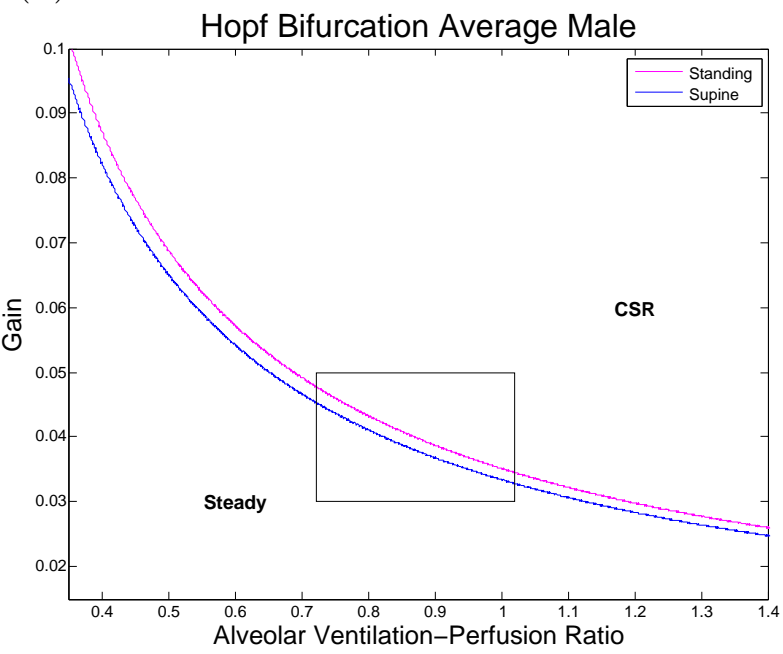

(d)

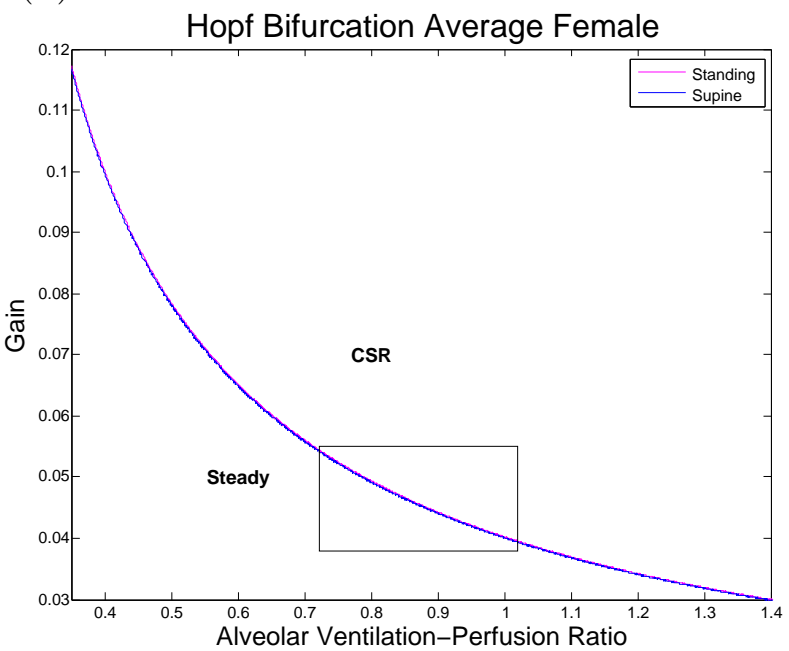

Figure 5.11: (a), (b): male average. (c), (d): female average. (b), (d): standing st $_{5}$ and supine $_{90}$. (a), (c): all body positions. Boxes represent typical $r$ and $\mu$ values. 


\section{Chapter 6}

\section{Conclusions and Future Work}

The models presented represent the cardiovascular, respiratory, and feedback control systems found in the human body. Steady state regions along with regions of oscillatory breathing are displayed using a Hopf bifurcation curve. It was predicted that obesity, body position, and gender would play key roles in determining an individual's likelihood of developing CSR. First, the model shows that obesity alone had minor effects on an individual's likelihood of developing CSR. Results were not strong enough to support our prediction. Next, the model suggests that body position (standing vs. supine) is a determining factor for CSR. Further, it shows that there is an increased incidence of CSR in the recumbent position compared to the upright position, which is in line with what we know since CSR primarily occurs during sleep [47]. Lastly, it is well known that CSR is more prevalent in males than females, however there was no concrete explanation. These models suggest that the discrepancies between male and female fluid shift provides support and a possible explanation for the irregularities known between genders.

Analysis of the models suggest that body fluid shift is the main determining factor linking all three predictions. Obesity is typically an indicator of fluid build up, change in body position shifts fluid throughout the body, and gender determines how the fluid will shift, each resulting in excess fluid buildup onto the neck. These shifts in body

fluid and fundamentally the amount of excess fluid on the neck has been shown in the 
model to be responsible for altering an individual's likelihood of developing apneas, hyperpneas, and oscillatory breathing; ultimately resulting in CSR.

Despite the results found regarding fluid shift, it should be noted that the gain is a larger determining factor than body fluid shift on altering the prevalence of CSR in an individual. Major changes in the value of the gain occur after patients suffer from congestive heart failure, a stroke, or atrial fibrillation [14, 47]. These alone would drastically increase the incidence of CSR in any individual compared to increases seen during body fluid shift.

The majority of future work for this model is founded in the biological data. To improve the model, differentiating between male and female parameter values will be of value, specifically the blood flow rate at rest. Increasing the number of subjects will allow results to be more accurate and generalizations will better be made for males and females. Further, all current subjects do not display any type of oscillatory breathing pattern (including CSR), and have not suffered from a stroke or heart failure. Thus introducing subjects with CSR could aid in determining how fluid shift, specifically obesity, body position, and gender, can change a subjects likelihood. Adaptations could also be made to the model to include age, height and BMI, as it is known that they are factors that affect the likelihood of developing CSR. 


\section{Appendix}

\section{Perfusion Constant}

This calculation was originally done in [15]; below is the full description.

The conversion of the transport of $\mathrm{CO}_{2}$, in volume units $(\mathrm{mL} / \mathrm{min}$ at $\mathrm{STP})$ in the blood, to units of partial pressure $(\mathrm{mmHg} / \mathrm{min})$ in the alveoli, is derived assuming that the ideal gas law holds in the alveoli [45]; that is $P V=n R T$, where $P$ is pressure, $V$ is volume, $n$ is the number of moles of the gas, $R$ is a constant, and $T$ is absolute temperature. In determining the volume of $n$ moles of a gas in the blood according to this law, the conventional assumption is that the gas is at standard temperature $T S=273^{\circ} \mathrm{K}$, standard pressure $P S=760 \mathrm{mmHg}$, and the gas is dry (free of water vapour); this is referred to as STPD [45]. In contrast, in the lungs we have a gas at body temperature $T B=310^{\circ} \mathrm{K}$, atmospheric pressure, and saturated with water vapour; this is referred to as BTPS [45]. In the blood, assuming STPD values the rate of change of $\mathrm{CO}_{2}$ by the ideal gas law is

$$
\dot{n}=\frac{P_{S}}{R T_{S}} \dot{V}
$$

where $\dot{V}$ is the rate in $\mathrm{mL} / \mathrm{min}$ STPD at which $\mathrm{CO}_{2}$ crosses the respiratory membrane and $\dot{n}$ is the rate in moles/min. Let $\dot{P}$ represent the rate of change in partial pressure of this same $\mathrm{CO}_{2}$ entering the alveoli. In the alveoli we assume $V=V_{9}, T=T_{B}$ and 
the ideal gas law gives the relation

$$
\dot{n}=\frac{V_{9}}{R T_{B}} \dot{P} .
$$

Since the amount of $\mathrm{CO}_{2}$ in moles leaving the blood equals the amount in moles entering the alveoli, we can eliminate $\dot{n}$ between (29) and (30) and find

$$
\dot{P}=\left(\frac{P_{S} T_{B}}{V_{9} T_{S}}\right) \dot{V} \equiv d \cdot \dot{V},
$$

which yields the required conversion constant $d=0.28767 \mathrm{mmHg} / \mathrm{mL}$.

\section{Appendix A: Congestive Heart Failure Model}

\begin{tabular}{ll}
\hline Quantity & Typical Value \\
\hline Blood flow rate at rest & $\dot{Q}=5 \mathrm{~L} / \mathrm{min}$ \\
Tidal volume per breath & $V_{T}=500 \mathrm{~mL}$ \\
\hline Alveolar ventilation rate at rest & $\dot{V}_{A}=4-5 \mathrm{~L} / \mathrm{min}$ \\
Alveolar gas volume & $V_{9}=3 \mathrm{~L}$ \\
\hline Inspired $C O_{2}$ partial pressure & $p_{I}=0.3 \mathrm{mmHg}$ \\
Metabolic $C O_{2}$ production at rest & $\dot{M}=200 \mathrm{~mL} / \mathrm{min}$ \\
Total volume of blood in body & $v_{T}=5 \mathrm{~L}$ \\
Pulmonary veins volume & $v_{1}=0.25 \mathrm{~L}$ \\
Left heart volume & $v_{2}=0.15 \mathrm{~L}$ \\
Aorta volume & $v_{3}=0.3 \mathrm{~L}$ \\
Main arteries volume & $v_{4}=0.5 \mathrm{~L}$ \\
Arterioles of the abdomen volume & $v_{5}=0.2 \mathrm{~L}$ \\
Systemic capillaries of the leg volume & $v_{6}=0.2 \mathrm{~L}$ \\
Systemic veins, pulmonary arteries, right heart volume & $v_{7}=3.3 \mathrm{~L}$ \\
Pulmonary capillaries volume & $v_{8}=0.1 \mathrm{~L}$ \\
Ventilation-perfusion ratio $\dot{\vec{V}}_{A} / \dot{Q}$ & $r=0.9$ \\
Cardiovascular efficiency $\dot{M} / \dot{Q}$ & $m=40 \mathrm{~mL} / \mathrm{L}$ \\
\hline
\end{tabular}

Table 6.1: Typical parameters for Dong and Langford model [15]. 


\begin{tabular}{llc}
\hline Quantity & Typical Value & References \\
\hline Blood flow rate at rest & $\dot{Q}=5 \mathrm{~L} / \mathrm{min}$ & {$[15,22,24,28]$} \\
Alveolar ventilation rate at rest & $\dot{V}_{A}=4-5 \mathrm{~L} / \mathrm{min}$ & {$[15,22,25,28]$} \\
Alveolar gas volume & $V_{G}=3 \mathrm{~L}$ & {$[15,21,22]$} \\
Inspired $\mathrm{CO}_{2}$ partial pressure & $p_{I}=0.3 \mathrm{mmHg}$ & {$[15,22]$} \\
Alveolar $\mathrm{CO}_{2}$ partial pressure at rest & $p_{9}=40 \mathrm{mmHg}$ & {$[15,22,25]$} \\
Metabolic $\mathrm{CO}_{2}$ production at rest & $\dot{M}=200 \mathrm{~mL} / \mathrm{min}$ & {$[15,24,42]$} \\
Perfusion constant & $d=0.28767 \mathrm{mmHg} / \mathrm{mL}$ & {$[6,15]$} \\
Ventilation-perfusion ratio $\dot{V}_{A} / \dot{Q}$ & $r=0.9$ & {$[6,15]$} \\
Cardiovascular efficiency $\dot{M} / \dot{Q}$ & $m=40 \mathrm{~mL} / \mathrm{L}$ & {$[15]$} \\
\hline
\end{tabular}

Table 6.2: Typical parameter values for fluid shift model.

\section{Appendix B: Fluid Shift Model}

\section{Physiological Parameters}

Total neck, chest, abdomen, and leg volumes are measured using bioelectrical impedance for each subject and recorded [37]. Results are displayed in Table 6.4. Breakdowns of each region in specific model compartments can be seen in Table 6.3. 


\begin{tabular}{|c|c|c|c|}
\hline Region & Compartment & Amount & Reference \\
\hline \multicolumn{4}{|l|}{ Neck } \\
\hline & Arterioles $_{N}$ & $5 \%$ & {$[15]$} \\
\hline & Carotid Artery, Arms & $13 \%$ & {$[15]$} \\
\hline & Systemic Capillaries $_{N}$ & $5 \%$ & {$[15]$} \\
\hline & Jugular Vein & $77 \%$ & {$[15]$} \\
\hline \multicolumn{4}{|l|}{ Thorax } \\
\hline & Pulmonary Veins & $16 \%$ & {$[15]$} \\
\hline & Left Heart & $9 \%$ & {$[15]$} \\
\hline & Aorta & $19 \%$ & {$[15]$} \\
\hline & Main Arteries & $31 \%$ & {$[15]$} \\
\hline & Pulmonary Arteries, Rt Heart & $19 \%$ & {$[15]$} \\
\hline & Pulmonary Capillaries & $6 \%$ & {$[15]$} \\
\hline \multicolumn{4}{|l|}{ Abdomen } \\
\hline & Arterioles $_{A}$ & $6 \%$ & {$[15]$} \\
\hline & Systemic Capillaries $_{A}$ & $6 \%$ & {$[15]$} \\
\hline & Systemic Veins $_{A}$ & $88 \%$ & {$[15]$} \\
\hline \multicolumn{4}{|l|}{ Legs } \\
\hline & Systemic Capillaries $_{L}$ & $6 \%$ & {$[15]$} \\
\hline & Arterioles $_{L}$ & $6 \%$ & {$[15]$} \\
\hline & Systemic Veins ${ }_{l}$ & $88 \%$ & {$[15]$} \\
\hline
\end{tabular}

Table 6.3: Breakdown of compartmental volumes from total.

\begin{tabular}{|c|c|c|c|c|c|c|}
\hline Subject & Gender & Position & $\begin{array}{c}\text { Leg } \\
(\mathrm{mL})\end{array}$ & $\begin{array}{l}\text { Abdomen } \\
(\mathrm{mL})\end{array}$ & $\begin{array}{l}\text { Chest } \\
(\mathrm{mL})\end{array}$ & $\begin{array}{c}\text { Head and } \\
\text { Neck }(\mathrm{mL})\end{array}$ \\
\hline \multirow[t]{2}{*}{ Subject 1} & \multirow[t]{2}{*}{$\mathrm{M}$} & Standing $_{5}$ & 2016 & 467 & 2302 & 215 \\
\hline & & Supine $_{90}$ & 1971 & 529 & 2207 & 293 \\
\hline \multirow[t]{2}{*}{ Subject 2} & \multirow[t]{2}{*}{ M } & Standing $_{5}$ & 3234 & 428 & 1171 & 167 \\
\hline & & Supine $_{90}$ & 2989 & 413 & 1392 & 206 \\
\hline \multirow[t]{2}{*}{ Subject 3} & \multirow[t]{2}{*}{$\mathrm{F}$} & Standing $_{5}$ & 2244 & 744 & 1689 & 323 \\
\hline & & Supine $_{90}$ & 1773 & 813 & 2035 & 379 \\
\hline \multirow[t]{2}{*}{ Subject 4} & \multirow[t]{2}{*}{$\mathrm{F}$} & Standing $_{5}$ & 2646 & 632 & 1426 & 295 \\
\hline & & Supine 90 & 2350 & 650 & 1654 & 345 \\
\hline \multirow{2}{*}{ Subject 5} & \multirow[t]{2}{*}{ F } & Standing $_{5}$ & 2053 & 552 & 2141 & 255 \\
\hline & & Supine $_{90}$ & 1742 & 600 & 2360 & 298 \\
\hline \multirow[t]{2}{*}{ Subject 6} & \multirow[t]{2}{*}{$\mathrm{F}$} & Standing $_{5}$ & 2270 & 940 & 1565 & 225 \\
\hline & & Supine $_{90}$ & 1888 & 961 & 1885 & 265 \\
\hline \multirow[t]{2}{*}{ Subject 7} & \multirow[t]{2}{*}{$\mathrm{F}$} & Standing $_{5}$ & 2642 & 880 & 1180 & 298 \\
\hline & & Supine $_{90}$ & 2277 & 935 & 1418 & 370 \\
\hline
\end{tabular}

Table 6.4: Normalized fluid volume of each subject standing and in the supine position. 


\section{Regional Blood Flow/Metabolic Rate at Rest}

The total blood flow rate at rest, $\dot{Q}$, is widely known as $5 \mathrm{~L} / \mathrm{min}[15,22,24,28]$. For this model it was necessary to subdivide $\dot{Q}$ into regional blood flow rates at rest. This was done by assigning a certain percentage of $\dot{Q}$ to the abdomen, legs, and neck. Cardiac output is the amount of blood pumped by the heart per minute [21]. Distributions based on body segments can be found in [21, 23, 32], which subdivide the total blood flow rate into regional rates, denoted $J$. The trunk, arms, and upper and lower legs were categorized and the skeletal muscle, bone, skin and other categories were broken down done based on the distributions found in [2]. Further, they were subdivided into male and female categories. Results can be seen in Table 6.5.

The metabolic rate throughout the body is broken down into rates in different tissues and organs in $[21,44]$. It is not divided by male or female.

\begin{tabular}{lll}
\hline Parameter & Quantity \\
& Male & Female \\
\hline Abdomen blood flow fraction & $J_{A}=0.6662$ & $J_{A}=0.6594$ \\
Leg blood flow fraction & $J_{L}=0.1598$ & $J_{L}=0.17$ \\
Neck blood flow fraction & $J_{N}=0.174$ & $J_{N}=0.1706$ \\
Abdomen metabolic $\mathrm{CO}_{2}$ production fraction & $K_{A}=0.81$ & $K_{A}=0.81$ \\
Leg metabolic $\mathrm{CO}_{2}$ production fraction & $K_{L}=0.01$ & $K_{L}=0.01$ \\
Neck metabolic $\mathrm{CO}_{2}$ production fraction & $K_{N}=0.18$ & $K_{N}=0.18$ \\
\hline
\end{tabular}

Table 6.5: A - abdomen. L - legs. $\mathrm{N}$ - neck. $J_{A}+J_{L}+J_{N}=1=K_{A}+K_{L}+K_{N}$. 


\section{Bibliography}

[1] Philip I. Aaronson, Jeremy P.T. Ward, Charles M. Wiener, Steven P. Schulman, and Jaswinder S. Gill. The cardiovascular system at a glance. Blackwell Science, 1999.

[2] T. Abe, C. F. Kearns, and T. Fukunaga. Sex differences in whole body skeletal muscle mass measured by magnetic resonance imaging and its distribution in young Japanese adults. British Journal of Sports Medicine, 37(5):436-40, January 2003.

[3] J.P. Adams and P.G. Murphy. Obesity in anaesthesia and intensive care. British Journal of Anaesthesia, 85(1):91-108, 2000.

[4] James K. Alexander. Obesity and the circulation. Modern Concepts of Cardiovascular Disease, XXXII(32/6):799-803, 1963.

[5] James K. Alexander, Edward W. Dennis, William G. Smith, Kamel H. Amad, Christopher Duncan, and Robert C. Austin. Blood volume, cardiac output, and distribution of systemic blood flow in extreme obesity. Cardiovascular Reasearch Center Bulletin, 1:39-44, 1962.

[6] Julie Atamanyk and William F Langford. A compartmental model of CheyneStokes respiration. Fields Insititute Communications, 36:1-16, 2003. 
[7] Frank Barrera, Marcus Reidenberg, William Winters, and Suwana Hungspreugs. Ventilation-perfusion in the obese patient relationships. Journal of Applied Physiology, 26(4):420-426, 1969.

[8] Jerry J. Batzel and Mostafa Bachar. Modeling the cardiovascular-respiratory control system: data, model analysis, and parameter estimation. Acta Biotheoretica, 58(4):369-80, December 2010.

[9] Jerry J. Batzel, Franz Kappel, Daniel Schneditz, and Hien T. Tran. Cardiovascular and respiratory system: modeling, analysis, and control. SIAM Frontiers in Applied Mathematics, 2007.

[10] Jerry J. Batzel, Susanne Timischl-teschl, and Franz Kappel. A cardiovascularrespiratory control system model including state delay with application to congestive heart failure in humans. Journal of Mathematical Biology, 335(50):293$335,2005$.

[11] H.T. Batzel, J.J. and Tran. Stability of the human respiratory control system. Journal of Mathematical Biology, 102(41):80-102, 2000.

[12] H. E. Berg, B. Tedner, and P. A. Tesch. Changes in lower limb muscle crosssectional area and tissue fluid volume after transition from standing to supine. Acta physiologica Scandinavica, 148(4):379-85, August 1993.

[13] T. Douglas Bradley and John S. Floras. Sleep apnea and heart failure: Part I: obstructive sleep apnea. Circulation, 107(12):1671-8, April 2003.

[14] T. Douglas Bradley and John S. Floras. Sleep apnea and heart failure: Part II: central sleep apnea. Circulation, 107(13):1822-6, April 2003. 
[15] Fang Dong and William F. Langford. Models of Cheyne-Stokes respiration with cardiovascular pathologies. Journal of Mathematical Biology, 57(4):497-519, October 2008.

[16] Karen E. Etter, Nandu Goswami, and M. Keith Sharp. Modelling of cardiovascular response to graded orthostatic stress: role of capillary filtration. European Journal of Clinical Investigation, 41(8):807-19, August 2011.

[17] Joseph Feldschuh and Yale Enson. Prediction of the normal blood volume. Relation of blood volume to body habitus. Journal of the American Heart Association, 56(4):605-612, 1977.

[18] Martin Fink, Jerry J. Batzel, and Franz Kappel. An optimal control approach to modeling the cardiovascular-respiratory system : An application to orthostatic stress. Cardiovascular Engineering, 4(1):27-38, 2004.

[19] A. C. Fowler and G. P. Kalamangalam. The role of the central chemoreceptor in causing periodic breathing. IMA Journal of Mathematics Applied in Medicine and Biology, 17(2):147-67, June 2000.

[20] D. P. Francis, K. Willson, L. C. Davies, A. J. S. Coats, and M. Piepoli. Quantitative general theory for periodic breathing in chronic heart failure and its clinical implications. Circulation, 102(18):2214-21, October 2000.

[21] Fred S. Grodins, June Buell, and Alex J. Bart. Mathematical analysis and digital simulation of the respiratory control system. Journal of Applied Physiology, $22(2): 260-76,1967$.

[22] John E. Hall and Arthur C. Guyton. Textbook of medical physiology. SAUNDERS Elsevier, Philadelphia, PA, 2011. 
[23] S. T. Hussain. Blood flow measurements in lower limb arteries using duplex ultrasound. Annals of the Royal College of Surgeons of England, 79(5):323-30, September 1997.

[24] Franz Kappel, Martin Fink, and Jerry J. Batzel. Aspects of control of the cardiovascular-respiratory system during orthostatic stress induced by lower body negative pressure. Mathematical Biosciences, 206(2):273-308, April 2007.

[25] James Keener and James Sneyd. Mathematical Physiology. Springer; Corrected edition (October 1, 1998), 1 edition, 1998.

[26] M. C. Khoo, R. E. Kronauer, K. P. Strohl, and A. S. Slutsky. Factors inducing periodic breathing in humans : a general model. Journal of Applied Physiology, 53:644-659, 1982.

[27] Prem Kumar and Nanduri R. Prabhakar. Peripheral chemoreceptors: Function and plasticity of the carotid body. In Comprehensive physiology. John Wiley \& Sons, Inc., 2011.

[28] G. S. Longobardo, N. S. Cherniack, and A. P. Fishman. Cheyne-Stokes breathing produced by a model of the human respiratory system. Journal of Applied Physiology, 21(6):1839-46, November 1966.

[29] M. C. Mackey and L. Glass. Oscillation and chaos in physiological control systems. Science, 197:287-289, 1977.

[30] Paul L. Marino and Kenneth M. Suttin. The ICU book. Lippincott Williams \& Wilkins, 2007.

[31] Howard T. Milhorn, Richard Benton, Richard Ross, and Arthur C. Guyton. A mathematical model of the human respiratory control system. Biophysical Journal, 5:27-46, 1965. 
[32] David E. Mohrman and Lois Jane Heller. Cardiovascular Physiology. McGraw Hill, 7 th edition, 2010.

[33] Jorge L. Moiola and Guanrong Chen. Hopf Bifurcation Analysis: A frequency domain approach. World Scientific Publinshing Co Pte Ltd, series a. edition, 1996.

[34] M. T. Naughton. Pathophysiology and treatment of Cheyne-Stokes respiration. Thorax, 53(6):514-8, June 1998.

[35] R. G. O'Regan and S. Majcherczyk. Role of peripheral chemoreceptors and central chemosensitivity in the regulation of respiration and circulation. The Journal of Experimental Biology, 100:23-40, October 1982.

[36] John L. Powell. Historical notes in pelvic surgery. Journal of Pelvic Surgery, $7(1): 37-38,2001$.

[37] Stefania Redolfi, Dai Yumino, Pimon Ruttanaumpawan, Brian Yau, Mao-chang Su, Jennifer Lam, and T. Douglas Bradley. Relationship between overnight rostral fluid shift and obstructive sleep apnea in nonobese men. American Journal of Respiratory and Critical Care Medicine, 179:241 - 246, 2009.

[38] C. Sahlin, E. Svanborg, H. Stenlund, and K. A. Franklin. Cheyne-Stokes respiration and supine dependency. The European Respiratory Journal : Official Journal of the European Society for Clinical Respiratory Physiology, 25(5):82933, May 2005.

[39] Ruchi Singhal and V. K. Katiyar. Mathematical models of Cheyne-Stokes breathing in understanding cardiovascular and respiratory disorders. Indian Journal of Biomechanics, 7-8(March):170-174, 2009. 
[40] Mao-Chang Su, Kuo-Liang Chiu, Pimon Ruttanaumpawan, Satomi Shiota, Dai Yumino, Stefania Redolfi, James S. Haight, Brian Yau, Jennifer Lam, and T. Douglas Bradley. Difference in upper airway collapsibility during wakefulness between men and women in response to lower-body positive pressure. Clinical Science, 116(9):713-20, May 2009.

[41] Joan Carles Trullàs, Francesc Formiga, Manuel Montero, Margarita CarreraIzquierdo, Jordi Grau-Amorós, David Chivite-Guillén, and Luís Manzano. Impact of weight loss on mortality in chronic heart failure: Findings from the RICA Registry. International Journal of Cardiology, October 2012.

[42] B. Vielle and G. Chauvet. Mathematical study of periodic breathing as an instability of the respiratory system. Mathematical Biosciences, 114(2):149-72, April 1993.

[43] P. D. Wagner, R. B. Laravuso, R. R. Uhl, and J. B. West. Continuous distributions of ventilation-perfusion ratios in normal subjects breathing air and 100 per cent O2. The Journal of Clinical Investigation, 54(1):54-68, July 1974.

[44] Zimian Wang, Zhiliang Ying, Anja Bosy-westphal, Junyi Zhang, Britta Schautz, Wiebke Later, Steven B. Heymsfield, and Manfred J. Mu. Specific metabolic rates of major organs and tissues across adulthood : evaluation by mechanistic model of resting energy expenditure 1 4. The American Journal of Clinical Nutrition, 92(4):1369-77, 2010.

[45] John B. West. Respiratory Physiology - The Essentials. Williams and Wilkins, 1974.

[46] M. J. Yiengst and N. W. Shock. Blood and plasma volume in adult males. Journal of Applied Physiology, 17:195-8, March 1962. 
[47] D. Yumino and T. D. Bradley. Central Sleep Apnea and Cheyne-Stokes Respiration. Proceedings of the American Thoracic Society, 5(2):226-236, February 2008. 